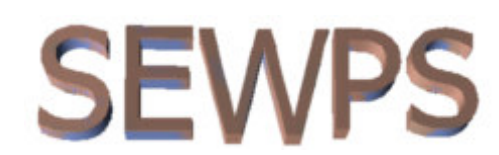

SPRU Electronic Working Paper Series

\author{
Paper No. 134
}

\title{
Indicators for Complex Innovation Systems
}

\author{
J. Sylvan Katz \\ (SPRU)
}

\section{May 2005}

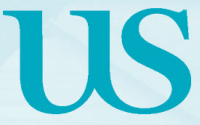

University of Sussex

SPRU - Science \& Technology Policy Research
The Freeman Centre, University of Sussex, Falmer, Brighton BN1 9QE, UK Tel: +44 (0) 1273678175 E-mail: j.s.katz@sussex.ac.uk http://www.sussex.ac.uk/spru/ 


\title{
Indicators for Complex Innovation Systems
}

\author{
Prepared by \\ J. Sylvan Katz \\ SPRU, \\ The Freeman Centre, \\ University of Sussex, \\ Brighton, East Sussex, UK BN1 9QE \\ j.s.katz@sussex.ac.uk
}

This work was supported by a grant from the Common Basis for Science, Technology and Innovation Indicators part of the Improving Human Research Potential specific programme of the Fifth Framework for Research and Technological Development" of the European Commission. It is part of the WISER project (Web indicators for scientific, technological and innovation research) (Contract HPV2-CT-2002-00015)."

\section{May 2005}




\begin{abstract}
Innovation systems are complex systems that can exhibit scaling and emergent properties. Predictable and measurable scaling correlations exist between measures commonly used to characterize innovation systems and national economies.

This paper examines scaling relationships between GERD \& GDP and between GDP \& population and uses them to construct scale-independent indicators of the European and Canadian innovation systems. It discusses the theory and practice of building scaleindependent indicators and scale-independent models. The theory is based on knowledge gathered from the study of complex systems. The practice is illustrated using OECD and Statistics Canada data commonly used to construct conventional indicators.
\end{abstract}

Keywords: complex system, scaling, power law, emergent properties, innovation, innovation system, indicators, scale-independent, model 


\section{Introduction}

Observers of innovation systems make comparisons (Freeman, 1987; Katz et al., 1998; Lundvall, 1992; Stoneman, 1995). Invariably they compare groups or collective entities such as countries, institutions, departments, firms, etc. over time and often broken down by scientific or technology field. They use quantitative and qualitative measures of the inputs, outputs and processes in the systems to construct indicators that frequently inform government programs and public policy.

According to the OECD a science and technology indicator is

a series of data which measures and reflects the science and technology endeavour of a country, demonstrates its strengths and weaknesses and follows its changing character notably with the aim of providing early warning of events and trends which might impair its capability to meet the country's needs (Godin, 2005; OECD, 1976).

This paper will focus only on quantitative indicators. A quantitative indicator is defined as numbers or ratios of numbers derived from empirical ${ }^{1}$ observations that can be used to inform public policy.

There is a wide variety of indicators ${ }^{2}$. For example, a standard measure of the size of an economy is its Gross Domestic Product (GDP). It is an indicator of the market value of goods and services from all sectors in the economy. Similarly the gross expenditure on research and development (GERD) is a cherished indicator of a country's R\&D effort (Godin, 2005). GERD, expressed as a percentage of GDP, is commonly called the R\&D intensity indicator. It is frequently used to compare the R\&D effort of innovation systems.

${ }^{1}$ Empirical means information based on experience or observational information and not necessarily on proven scientific data. US NIH glossary http://www.niaid.nih.gov/factsheets/GLOSSARY.htm

2 In this paper a distinction will not be made between science and technology indicators and knowledge and innovation indicators commonly used to measure and evaluate innovation systems and their members. 
Often, a government will use the R\&D intensity as a target in its policy objectives to argue for more R\&D resources (Voyer, 1999).

Another commonly used indicator is the number of research documents published by the scientists and engineers in an innovation system. It is used as a measure of the size of the system (Katz et al., 1998). A variety of measures such as the number of firms or scientists and engineers are also used as indicators of the size of an innovation system. The impact of a country's published output is frequently measured by the number of citations to its publications. Sometimes, groups are compared using indicators based on numbers of citations per paper (A.F.J Van Raan, 2005). It is best practice for a group of peers to review a collection of indicators to see if they converge on program or policy relevant issues (Irvine et al., 1983).

Since the official beginning of the use of S\&T indicators by the OECD in the 1960s many warnings have been made about employing indicators for comparative purposes (Godin, 2005). For example, as early as 1967 the OECD cautioned about the use of GERD for comparative purposes because "the percentage of GNP devoted to R\&D varies directly with per capita GNP. [But] this appears to be true at the top and bottom of the scale" (Godin, 2005). Furthermore warnings have been issued that international comparisons of R\&D expenditures are influenced by the scale of the economies being compared (Holbrook, 1991) The journal impact factor (Adams, 2002) and indicators based on citations/paper have been similarly criticized as misleading (Katz, 2000, 2005).

Many important indicators are derived from the ratio of common measures. For example, GDP per capita (GDP/population) is frequently used as an indicator of a nation's income and as previously mentioned GERD/GDP is used as an indicator of the R\&D intensity of an innovation system. It will be shown that GERD can exhibit a predictable scaling relationship with GDP and that GDP can show a predictable scaling relationship with population. Furthermore, it will be shown that when a scaling relationship exists between two measures (e.g. GERD/GDP, GDP/population and citations/paper) then the ratio of those measures also exhibits a scaling relationship. It will be claimed that these scaling relationships should be taken into account when comparisons are made between groups of different sizes. Indicators that have been adjusted for size or scale are called scaleindependent indicators (Katz, 1999, 2000, 2005). 
Van Raan (Anthony F. J. Van Raan, 2005 (submitted)) claims that "one could also argue that a larger impact as measured on the bases of citations cannot be simply waved aside as purely a scale-dependent effect. In this way groups are 'punished' for having reached a considerable size as the number of citations received by them should be corrected for $s^{3} e^{3 "}$. It will be argued that innovation systems are complex systems that exhibit emergent properties and they tend to scale. And it will argue that unless observers and policy-makers account for the non-linear scaling effects then their expectations of the performance of individual members of the system may be unrealistic.

This paper focuses on the theory and practice for constructing scale-independent indicators. The theory underlying these indicators is based on information gathered from the study of complex systems. The practice for constructing scale-independent indicators is illustrated using common statistical measures for two innovation systems, the European and Canadian innovation systems.

\section{Complex Systems, Scaling and Power Laws}

It is difficult to precisely define a complex system; however, it is recognizable because it has identifiable characteristics (Amaral et al., 2004). Amongst other things a complex system

- has a dynamic internal structure that evolves and interacts in a complex manner,

- exhibits emergent behaviours and patterns that are not the result of rules or caused by a single entity in the system. Flocking of birds, swarming of bees, schooling of fish and swirling of hurricanes are emergent properties found in nature (Parrish et al., 2002; Peterson, 2000). The stock market is a system that has emergent properties determined by the collective actions of investors (Blok, 2000).

- is open in the sense that information flows across its boundaries which in turn are difficult to clearly identify.

- is composed of complex subsystems.

${ }^{3}$ If Van Raan's argument were valid then there would be no reason to normalize the impact by dividing citations by number of papers. One purpose of normalization is to adjust for size so impact comparisons can be made between large and small groups. However, the current normalization procedure is only valid when there is a linear scaling relationship between citations and papers but this rarely occurs (Katz, 2005). 
Frequently a complex system is represented as a complex network with nodes representing the units and edges representing the interactions between them (Albert et al., 2002; Steven H. Strogatz, 2001). Researchers have developed a variety of models to simulate complex systems. Indicators commonly used to compare innovation systems have been used to confirm that many important properties exhibited by these models can be seen using empirical data (Albert et al., 2002; Amaral et al., 2001; Havemann et al., 2005). Examples of these will be discussed later.

Most, if not all, complex systems have at least one common feature. They have a propensity to exhibit scaling properties (Carlson et al., 2002; M. E. J. Newman, 2000). In other words they exhibit characteristics that are statistically similar at many levels of aggregation. For example, the swirl of a hurricane contains many smaller swirls and similarly a swarm of bees contains smaller swarms. The identifying signature of a scaling property is a power law relationship. Power laws are common to physical (Christensen et al., 2002; Warhaft 2002), natural science (Goldberger et al., 2002; Katz et al., 1999; West et al., 2002) and social systems (M. E. J. Newman et al., 2002). They describe well-known statistical regularities ${ }^{4}$ such as Pareto, Lotka, Bradford and Zipf's laws. These laws have been shown to hold for such things as the distribution of the sales and size of firms, the productivity of researchers and inventors, the journals in scientific fields and the use of language. These laws typify many characteristics of innovation systems (De Solla Price, 1963).

Formally a power law relationship is defined by $F(x) \propto x^{\alpha}$ were the variable of interest $x \in\left[x_{0}, x_{n}\right]$ and $\mathrm{n}>0$. In real world systems the range of a power law distribution may be finite since the tail of the distribution asymptotically approaches a power law as $\mathrm{x}$ gets large (Stanley et al., 2001). In other words, in real systems the range of an ideal power law relationship maybe constrained to a finite range. Power laws are readily identifiable when they are plotted on a log-log scale because they appear linear.

${ }^{4}$ Observers of social systems sometimes refer to these statistical regularities as social laws. 
The exponent, $\alpha$, of a power law is called its scaling factor and it is given by the slope of the linear regression line drawn through the log values. It is a useful indicator. For example, the area and the volume of a circle and a sphere increase as the square and cube of the radius, respectively. The scaling factor for a circle is 2.0 and for a sphere it is 3.0 and it tell us that if the radius doubles then the area of the circle increases four fold $\left(2^{2}\right)$ and the area of the sphere by eight fold $\left(2^{3}\right)$. The area of a circle and the volume of a sphere are emergent properties seen in many two and three dimensional objects. However, they are unique because the scaling factors of these emergent properties are integers. Scaling properties in most natural and social systems do not have integer scaling factors. For example, objects like clouds, plants and the World Wide Web can have scaling properties that have scaling factors between 1.0 and 3.0 (S. H. Strogatz, 2005).

If innovation systems are truly complex we would expect them to exhibit scaling and emergent properties. In fact, the tell-tale power law signatures of scaling relationships have been seen in innovation system indicators for decades. This is witnessed by the large number of papers that have been written describing the importance of Lotka, Pareto and Zipf distributions in the activities of parts of innovation systems (Katz, 1999). Some these papers have suggested models to explain the power law generating processes that produce scaling characteristics.

\section{Power Law Generators}

Power laws are generated by a wide variety of mechanisms (Mitzenmacher, 2003) that range from completely deterministic processes (strictly rule based) to completely nondeterministic processes (stochastic or random). In fact, Mitzenmacher says "Power law distributions and lognormal distributions are quite natural models and can be generated from simple and intuitive generative processes." Examples of three types of generative models will be discussed: (1) deterministic generators; (2) non-deterministic generators; and (3) mixed deterministic and non-deterministic generators.

\subsection{Deterministic generators}

Ideal exponential growth is deterministic since all past and future values are predictable. It can be shown that a pair of exponential processes that are coupled through a common variable such as time will exhibit a power law correlation where the scaling factor is given 
by the ratio of the exponents of the exponential processes ${ }^{5}$. This relationship was used to demonstrate scaling correlations between the growth of citations ${ }^{6}$ and papers in the ISI database (Katz, 2005). Deterministic power law generators have been identified that generate scale free networks (Barabasi et al., 2001; Dorogovtsev et al., 2001).

\subsection{Non-deterministic generators}

Brownian motion, the random motion of liquid and gas molecules, is an example of a non-deterministic generator of power law distributions. Stock market processes and gains \& losses from gambling activities generate brown noise which is an allusion to Brownian motion because both have a $1 / \mathrm{f}^{2}$ power spectrum distribution. Brownian motion contains several subtle statistical self-similarities or power laws distributions (Blok, 2000; Schroeder, 1991).

A number of models have been proposed to generate the power laws commonly seen in word frequency distributions of language. All but one of these models is based on a mixed generator (a random process plus one or more rules). This type of generator will be discussed in the next section. In 1957, a researcher proposed a model based on a monkey typing randomly on a keyboard. The characters were struck with equal probabilities. It has been mathematically proven that this model generates rank word frequency power law distribution (Mitzenmacher, 2003).

\subsection{Mixed generators}

Mixed deterministic and non-deterministic processes can be generators of power law distributions. A random multiplicative process is known to generate a lognormal or Gibrat distribution (Gibrat, 1931). This process is defined by $X_{t}=F_{t} X_{t-1}$ where $\mathrm{X}_{0}$ and $\mathrm{F}_{0}$ are the starting size and the initial growth factor. The growth factor can be positive or

${ }^{5}$ Assume we are given any two exponential processes $x=a e^{p t}$ and $y=b e^{q t}$. Using these two relationships $e=(x / a)^{1 / p t}=(y / b)^{1 / q t}$ and thus $(x / a)^{1 / p}=(y / b)^{1 / q}$ therefore it can be seen that $y=(b / a)^{q / p} x^{q / p}$ which has the form of $F(x) \propto x^{\alpha}$. In other words, any pair of coupled exponential processes will exhibit a power law correlation with exponent, $\alpha=q / p$, and intercept, $s=(b / a)^{q / p}$, that are predictable from the exponents and intercepts of the individual exponential processes. This relationship holds even if the two processes are delayed in time with respect to each other or if they have different starting values at $t=0$.

${ }^{6}$ Citations were counted using a 3 year window. 
negative representing growth and shrinkage (negative growth). However, if a random multiplicative process is bounded by a minimum then it will yield a power law distribution instead of a lognormal distribution (Mitzenmacher, 2003; Solomon et al., 1997). There is a wide variety of mixed power law generators with dynamics that are governed by random processes and one or more rules.

The chaos game (Barnsley, 1988) is a simple example. It involves a random number generator and a simple rule. A player starts playing by placing three points on a piece of paper and selecting an arbitrary starting point. Randomly one of the three points is chosen and a rule is applied. The rule is 'go half way from the current point to the randomly selected point and make a point at that position'. After thousands of steps the well-known features of the Sierpinski triangle or gasket fractal will be visible in the structure of the dots. The probability distribution of the sizes of the triangles in the Sierpinski triangle follows a power law (Schroeder, 1991).

Recent research has demonstrated that the standard deviation of the growth rate of firms has a scaling correlation with the size of the firms measured using sales (Amaral et al., 1998; Amaral et al., 2001). Furthermore, the scaling relationship remained whether size was measured using the number of employees, assets, costs of goods sold and plants, property or equipment. A power law correlation has also been found between the standard deviation of growth rates and the sizes of countries measured using GDP and between the standard deviation of growth rates of universities and their sizes measured using papers, patents and R\&D expenditure (Plerou et al., 1999).

Amaral et al. (2001) built a model to explain the scaling relationships. The model is based on three assumptions ${ }^{7}$ : (1) firms (groups) tend to organize into multiple divisions (subgroups) once they achieve a certain size; (2) there is a broad distribution of minimum size in the economy and (3) the growth rates of different divisions are independent of one another. In the model the growth rate of the size of each division in the firms evolved according to a random multiplicative process. New divisions were

${ }^{7}$ Many firms stumble at the point where they make divisions because they fail to appreciate the need for interdivision coordination or they micromanage too closely the divisions from the centre. Thus, growth rates are highly irregular. Source: Private communications with Cooper Langford, University of Calgary. 
formed and small divisions absorbed according to a well-defined lower bound and two fixed probabilities. The authors concluded that "the model predicts that the number of subunits comprising an organization and the typical size of these subunits obey scaling laws".

The web has spawned a variety of research activities some of which focus on constructing models to explain the evolution of its structure. A preferential attachment model has gained considerable favor because it appears to explain frequently observed scaling characteristics. Many investigators have reported that the probability distribution of in-links and out-links to web pages follow power law distributions (Albert et al., 2002; Barabasi et al., 1999; Faloutsos et al., 1999). The preferential model assumes that the web grows by continuously adding new nodes. The links between nodes are added in a preferential manner. The preference is determined by the popularity of web pages measured by the number of in-links. In other words, pages that are linked to more frequently are preferred over other pages. At each step in the model a new page is created and then an existing page is randomly selected. The probability that the new page will link to the existing page is determined by the number of in-links to the existing page. Over time the in-link probability distribution of the web that is generated by the model will be a power law.

In summary, a variety of processes generate power law distributions. Unlike some physical processes social activities are never completely deterministic nor are they completely random. Human activity is complex ranging from the free will of individuals to the laws of society. It is likely that most, if not all, of the power law distributions and correlations observed in complex social systems are generated by mixed processes. In the next section it will be shown that regional and national innovation systems exhibit scaling behaviors that (1) emerge with time and (2) exist at points in time. In both instances the scaling relationships are likely generated by complex mixed generators.

\section{Scaling and Innovation Systems}

An innovation system is a social construct. Its character emerges from the interactions between its members and the members of other systems. Some of the interactions are more "rule-like" than others because they are governed by laws, regulations, treaties, etc. Other interactions are more random because they are governed by complex social, 
political and economic forces. It seems reasonable to assume that most scaling relationships seen in an innovation system are produced by mixed generators.

If scaling relationships and emergent properties are prevalent in innovation systems they should be visible using data commonly used to measure their properties. The following examples explore scaling correlations between GERD \& GDP and GDP \& population for the European (EU15) and Canadian innovation systems (1) over time and (2) at points in time. Scale-independent indicators derived from these scaling relationships are used to explore emergent properties of the two innovation systems.

A scale-independent indicator is an indicator derived from a power law distribution or correlation. The phrase scale-independent is used because indicators that have been derived from a power law are normalized by the scaling relationship so they can be comparable over a wide range of sizes. This paper focuses on only two types of scaleindependent indicators: scaling factor indicators and relative magnitude indicators. Examples of both of these indicators will be given later.

There are other scale-independent indicators. For example, the distribution pattern of the data points about an ideal power law can provide indicators to underlying dynamics 8 (Katz et al., 1999). Sometimes the intercept of a power law is used as an indicator, particularly in physical systems. Also, some power law distributions have exponential cut-off points (Mossa et al., 2002; Mark E. J. Newman, 2001) that may be a useful indicator.

The European and Canadian innovation systems are used in this paper for two reasons. First, there is a large difference between the scales of the systems; by almost any measure the European system is about an order of magnitude larger than the Canadian system. Second, the structures of the two systems are considerably different. The European innovation system is a collection of fifteen national systems that has been evolving for about 50 years into a supranational system through a variety of democratic and legal processes (Schuch, 1998). All fifteen countries did not join at once. The European Economic Community was formed in 1958 and it consisted of 6 countries

${ }^{8}$ For example, the common patterns seen in European and Canadian data presented in Figure 7 might be indicative of a common dynamic. 
(Germany, France, Italy, the Netherlands, Luxembourg, and Belgium). The UK, Ireland and Denmark joined in 1973, Greece in 1981, Spain and Portugal in 1986 and Austria, Finland and Sweden in 1995. In contrast the Canadian innovation system is composed of ten provincial and two territorial systems that has evolved over about 135 years into a federal innovation system (StarMap, 2004). Most of the provinces had joined the Canadian Federation by 1873 . The last provinces to join were Saskatchewan and Alberta in 1905 and Newfoundland in 1949.

Funding for publicly funded research in the Canadian system comes primarily from the federal government with smaller contributions from the provincial and territorial governments. In contrast publicly funded research in the European system is funded primarily by national governments with smaller contributions from the European Commission. EC programmes, such as European Framework Programmes have a strong focus on activities that encourage more cohesion in the European research area. Similarly, in Canada a variety of government programs encourages federal cohesion in the Canadian innovation system.

The data in the following examples were sourced from the OECD and Statistics Canada. The Canadian data is more complete than OECD data. For example, GERD data is available for every Canadian province for every year in the time interval while the OECD data is missing certain values for many European nations. For analysis purposes missing European data was interpolated ${ }^{9}$. The economic data for the Canadian system are in a common currency and the OECD data has been converted to purchasing power parity at current prices in US dollars (PPP \$US). The conversion introduces errors into the OECD data (Neary, 2005) that are not found in the Canadian data. This can affect the quality of the indicators built from OECD data.

\subsection{Scaling over Time}

Figure 1 plots the growth of GDP and GERD from 1981 to 2000 for the European Union and Canada. Figure 1a shows that the European GDP tended to grow exponentially over time with an exponent of $0.051 \pm 0.001$. Over the same period the GERD also tended to

${ }^{9}$ The exponential growth trend over the time period was used to interpolate missing GERD values. 
grow exponentially with an exponent of $0.052 \pm 0.002$. Figure $1 \mathrm{~b}$ shows similar exponential growth trends in Canada.

[Figure 1 here]

It is apparent from the graphs that neither the GDP nor GERD exhibited perfect exponential growth. In fact we won't expect the growth to be perfectly exponential since the magnitude of the national and provincial GERDs and GDPs are determined by the interplay of many factors. On the other hand the exponential growth tendencies suggest there are some rule-like tendencies such as interest rates that exist in these systems. The mathematical relationship given in the footnote of 3.1 shows that two coupled exponential processes will exhibit a scaling correlation. GERD and GDP are coupled in time therefore they should exhibit a predictable scaling correlation. Using the values for the exponential growths from Figure 1 it is predicted that the scaling factor for the power law correlation between GERD and GDP for the European innovation system should be $0.052 / 0.051=1.027$. Figure $2 \mathrm{a}$ shows that the measured value was $1.034 \pm 0.028$ which is within $1 \%$ of the predicted value. The predicted value for the Canadian innovation system was $0.076 \pm 0.003 / 0.053 \pm 0.002=1.418$ and the measured value was $1.418 \pm$ 0.028 (Figure 2b).

[Figure 2 here]

When two measures exhibit a scaling relationship the ratio between those measures also exhibits a scaling relationship with the divisor. Consider a power law relationship given by $y=k x^{\alpha}$ then $y / x=k x^{\alpha-1}$. If a scaling relationship exists between GERD and GDP then GERD/GDP, the R\&D intensity indicator, should exhibit a scaling relationship with GDP. Using this relationship the R\&D intensity indicator for the European innovation system is predicted to scale with GDP with a scaling factor of 0.027 and the Canadian innovation system with a scaling factor of 0.418 . The measured values were $0.034 \pm$ 0.028 and $0.418 \pm 0.028$, respectively. Since the European GERD scaled nearly linearly with GDP its R\&D intensity almost remained constant over the time interval illustrated by 
the fact that its scaling factor was close to zero ${ }^{10}$. On the other hand the R\&D intensity for Canadian innovation system exhibited a tendency to increase 1.33 times $\left(2^{0.418}\right)$ when the GDP doubled. This is a strong non-linear tendency.

What do the scaling factors tell us about these two innovation systems? The GERD-GDP scaling factor tells us two things. The sign of the scaling factor indicates whether the GERD is growing faster or slower than GDP. The magnitude of the scaling factor indicates how much the GERD would be expected to grow as GDP increases. For example, over the 20 year period the European GERD tended to grow $2.05\left(2^{1.034}\right)$ times and the Canadian GERD tended to grow $2.67\left(2^{1.418}\right)$ times every time the GDP doubled $\left(2^{1.0}\right)$. In other words, the European GERD grew almost linearly with GDP and the Canadian GERD grew quite nonlinearly with GDP. This is supported by the fact that the OECD reported that the R\&D intensity for the European innovation system grew from $1.67 \%$ in 1981 to $1.89 \%$ in 2000 for a difference of $0.22 \%$. On the other hand the Canadian system grew from $1.24 \%$ in 1981 to $1.92 \%$ in 2000 for a larger difference of $0.68 \%$.

In summary, the scaling factor, $\alpha$, can be used as a scale-independent indicator. In the example it was used as a measure of the relative growths of two coupled exponential processes. When $\alpha=1$ then the relative growth rates are the same; when $\alpha>1$ then GERD is growing faster than GDP; and when $\alpha<1$ then GERD is growing slower than GDP.

The scaling factor will be used as an indicator in other examples. A naming notation is used to uniquely identify the scaling factor between the two variables, $X$ and $Y$ where variable $\log Y$ is regressed on variable $\log X$. The name given is the $Y-X$ scaling factor. For example in the previous example the indicator was called the GERD-GDP scaling factor because it compared the growth rate of GERD to GDP.

Inter-innovation system scale-independent indicators can also be produced. For example, since the European and Canadian systems exhibited exponential GDP growth trends and they are coupled in time they exhibit a scaling correlation. The scaling

${ }^{10}$ Only in the special case where $\alpha=1$, that is the relationship is linear, does $y / x=k$. 
relationship between the Canadian GDP $\left(\mathrm{GDP}_{\mathrm{C}}\right)$ and the European GDP (GDP $\left.\mathrm{E}_{\mathrm{E}}\right)$ had a $G_{C P}-G D P_{E}$ scaling factor equal to $1.05 \pm 0.03$. The $G E R D_{C}-G E R D_{E}$ scaling factor had a value of $1.43 \pm 0.03$. These indicators show that the Canadian GDP and GERD grew faster than the European GDP and GERD between 1981 and 2000. According to these scaling relationships if the European GDP and GERD doubled the Canadian GDP and GERD would be expected to increase $2.07\left(2^{1.05}\right)$ and $2.7\left(2^{1.43}\right)$ times, respectively.

Table 1 and Table 2 give the GERD-GDP scaling factors for the nations and provinces in the European and Canadian innovation systems. Also, the population in the year 2000 is given for each nation and province to give the reader a sense of their sizes.

Table 1 - European GERD-GDP Scaling Factors (1981-2000)

\begin{tabular}{lrccc}
\hline Country & $\begin{array}{r}\text { Pop. } \\
\text { ('000) }\end{array}$ & $\mathbf{\alpha}$ & $\mathbf{s e}^{*}$ & $\mathbf{R}^{\mathbf{2}}$ \\
\hline Austria & 8,012 & 1.50 & \pm 0.03 & 0.99 \\
Belgium & 10,246 & 1.26 & \pm 0.03 & 0.99 \\
Denmark & 5,338 & 1.82 & \pm 0.02 & 1.00 \\
Finland & 5,176 & 2.05 & \pm 0.05 & 0.99 \\
France & 60,594 & 1.11 & \pm 0.04 & 0.98 \\
Germany & 82,188 & 0.87 & \pm 0.05 & 0.95 \\
Greece & 10,917 & 2.58 & \pm 0.06 & 0.99 \\
Ireland & 3,799 & 1.49 & \pm 0.05 & 0.98 \\
Italy & 57,762 & 1.11 & \pm 0.09 & 0.91 \\
Netherlands & 15,922 & 1.03 & \pm 0.04 & 0.98 \\
Portugal & 10,225 & 1.84 & \pm 0.05 & 0.99 \\
Spain & 39,927 & 1.75 & \pm 0.06 & 0.98 \\
Sweden & 8,872 & 1.57 & \pm 0.05 & 0.98 \\
United Kingdom & 58,643 & 0.73 & \pm 0.02 & 0.99 \\
\hline
\end{tabular}

${ }^{*}$ se is the standard error for $\alpha$ 
Table 2 - Canadian GERD-GDP Scaling Factors (1981 - 2000)

\begin{tabular}{lrccc}
\hline Province & $\begin{array}{r}\text { Pop. } \\
\text { ('000) }\end{array}$ & $\mathbf{\alpha}$ & $\mathbf{s e}^{*}$ & $\mathbf{R}^{\mathbf{2}}$ \\
\hline Alberta & 3,010 & 1.09 & \pm 0.08 & 0.91 \\
British Columbia & 4,060 & 1.40 & \pm 0.06 & 0.97 \\
Manitoba & 1,146 & 1.06 & \pm 0.08 & 0.92 \\
New Brunswick & 756 & 1.38 & \pm 0.14 & 0.85 \\
Newfoundland \& Labrador & 538 & 1.21 & \pm 0.09 & 0.90 \\
Nova Scotia & 942 & 1.14 & \pm 0.07 & 0.94 \\
Ontario & 11,698 & 1.33 & \pm 0.04 & 0.98 \\
Prince Edward Island & 138 & 1.09 & \pm 0.07 & 0.94 \\
Quebec & 7,382 & 1.84 & \pm 0.05 & 0.99 \\
Saskatchewan & 1,022 & 1.35 & \pm 0.12 & 0.88 \\
\hline
\end{tabular}

${ }^{*}$ se is the standard error for $\alpha$

The standard errors and the $\mathrm{R}^{2}$ values indicate that the power law correlations have statistical significance. There appears to be a tendency for the significance to decrease slightly with the size of the nation or province measured by population. The GERDs for two of the largest nations in the European system, UK and Germany, did not grow as fast their respective GDPs. On the other hand the GERDs of Canadian provinces grew faster than their respective GDPs. The tables will be referred to again in later analysis.

\subsection{Scaling at Points in Time}

Figure $3 a$ is a log-log plot of 1990 GERD \& GDP values for nations in the European innovation system. 1990 was chosen because it is half way through the time interval under consideration. Figure $3 \mathrm{~b}$ is a similar plot for the provinces in the Canadian innovation system. The following question is being asked of these data. In 1990 did the members of the European and Canadian innovation systems exhibit a scaling correlation between GERD and GDP?

To help answer the question three regression lines have been drawn through the data points. There are two dotted lines and a solid line. The upper dotted line is a linear regression constrained to pass through the origin. This case is a special power law where the scaling factor is equal to 1.0. The lower dotted line is a linear regression that was not constrained to pass through the origin. The solid line is the power law regression line. The $\mathrm{R}^{2}$ statistics suggests that both linear regressions fit the data better than the 
power law. However, a visual inspection ${ }^{11}$ reveals that neither linear regression fits the data very well. The variances of the actual GERD values from the values predicted by the linear regressions vary with the size of the GDP. In other words, the data are heteroscedastic ${ }^{12}$ and the $R^{2}$ value has little statistical significance. On the other hand, the power law regression is close to homoscedastic and it has a good $R^{2}$ value. The same observations are true for the Canadian innovation system (Figure $3 b$ ).

\section{[Figure 3 here]}

This GERD-GDP scaling factor will be called a systemic scaling factor because it quantifies the relationship between GERD and GDP across members of the system at a point in time. The system scaling factor is not determined by any individual entity in the system. It evolves from the complex interaction between its members and between itself and other systems. It is an emergent property of a system.

The system GERD-GDP scaling factor tells us how the expenditures on R\&D by members of an innovation system tended to scale at a point in time with the size of the member economies. For example, the systemic GERD-GDP scaling factor for the European system was 1.25 telling us that when the size of the national economy doubles the systemic tendency was for GERD to increase by 2.4 times $\left(2^{1.25}\right)$. In the Canadian system GERD tended to increase by 2.2 times $\left(2^{1.13}\right)$.

As shown in the previous section the R\&D intensity is expected to show a tendency to scale with GDP. The measured scaling factor for the scaling relationship between R\&D intensity and GDP for the European innovation system in 1990 was $0.25 \pm 0.15$ and for the Canadian innovation system it was $0.13 \pm 0.04$. In other words, in the European innovation system the R\&D intensity showed a systemic tendency to increase 1.19 times $\left(2^{0.127}\right)$ with a doubling in country size measured by GDP. In the Canadian system R\&D intensity tended to increase 1.09 times $\left(2^{0.127}\right)$ as the province size doubled.

\footnotetext{
${ }^{11}$ The residuals were plotted against the estimated y values and it confirmed that the data were heteroscedastic.

${ }^{12}$ Data are heteroscedastic when the errors in the actual $y$ values from the predicted values are related to the size of $x$ values.
} 
The R\&D intensity indicator should be adjusted for scaling effects before using it to compare countries and provinces. For instance Austria and Saskatchewan are 1/10 the size of Germany and Ontario, respectively. The systemic scaling relationships between GERD and GDP for the European and Canadian innovation systems indicate that the R\&D intensity for Germany was expected to be about $75 \%$ higher than for Austria and the R\&D intensity for Ontario should be approximately $35 \%$ higher than for Saskatchewan ${ }^{13}$. A relative GERD indicator will be introduced shortly that can be used instead of the R\&D intensity indicator because it has been adjusted for the scaling relationship between GERD and GDP.

Some might argue that when the scaling factor is close to 1.0 the nonlinear effects can simply be ignored. Assume the scaling factor is 1.05 and that we wish to compare two innovation systems where one system has an order of magnitude larger GDP than the other system. Given a scaling factor of 1.05 we would expect the larger system to have a GERD 11.2 times $\left(10^{1.05}\right)$ larger than the smaller one. In other words, the larger system would be expected to have a $12 \%$ larger GERD than if we assumed the scaling factor was 1.0 or linear. Thus, a small scaling factor can have a large effect.

Figures $3 \mathrm{a}$ and $3 \mathrm{~b}$ shows a striking difference between the European and Canadian innovation systems. The national GERDs in the European system exhibit a larger range of variances from the GERDs predicted by the systemic scaling correlation than the range of variances displayed by the provincial GERDs from the Canadian systemic scaling correlation. This difference probably occurs because the European innovation system is more loosely coupled than federal Canadian system. It will be shown that the variances of the two systems evolve differently over time.

Table 3 and 4 give the relative GERD indicators for the members of each system in 1981, 1990 and 2000. The relative GERD indicator is calculated by taking the ratio between the actual GERD and the GERD predicted by the measured systemic scaling correlation. For example, Table 3 shows that the UK had a relative GERD of $1.57,1.13$ and 1.07 in 1981, 1990 and 2000, respectively. In other words, in 1981 the UK GERD was 1.57 times larger than the amount predicted by systemic scaling correlation between

${ }^{13}$ The percentages were determined by using the fact that $10^{0.25}=1.78$ and $10^{0.127}=$ 1.34 . 
GERD and GDP across members of the European innovation system in 1981. By the year 2000 the value had dropped to 1.07 .

Table 3 - European Relative GERD Indicators

\begin{tabular}{lccc}
\hline Country & $\mathbf{1 9 8 1}$ & $\mathbf{1 9 9 0}$ & $\mathbf{2 0 0 0}$ \\
\hline Austria & 1.32 & 1.17 & 1.18 \\
Belgium & 1.64 & 1.27 & 1.23 \\
Denmark & 1.39 & 1.47 & 1.37 \\
Finland & 1.63 & 1.79 & 2.08 \\
France & 1.22 & 1.21 & 1.26 \\
Germany & 1.39 & 1.26 & 1.43 \\
Greece & 0.20 & 0.30 & 0.41 \\
Ireland & 1.18 & 0.94 & 0.71 \\
Italy & 0.56 & 0.67 & 0.62 \\
Netherlands & 1.73 & 1.49 & 1.13 \\
Portugal & 0.39 & 0.46 & 0.49 \\
Spain & 0.33 & 0.50 & 0.55 \\
Sweden & 2.51 & 2.25 & 2.31 \\
United Kingdom & 1.57 & 1.13 & 1.07 \\
\hline
\end{tabular}

Table 4 - Canadian Relative GERD Indicators

\begin{tabular}{lccc}
\hline Province & $\mathbf{1 9 8 1}$ & $\mathbf{1 9 9 0}$ & $\mathbf{2 0 0 0}$ \\
\hline Alberta & 0.97 & 0.85 & 0.61 \\
British Columbia & 0.66 & 0.76 & 0.81 \\
Manitoba & 1.25 & 0.99 & 1.03 \\
New Brunswick & 0.78 & 0.98 & 0.70 \\
Newfoundland \& Labrador & 1.01 & 1.15 & 0.95 \\
Nova Scotia & 1.50 & 1.33 & 1.32 \\
Ontario & 1.29 & 1.05 & 1.27 \\
Prince Edward Island & 1.04 & 0.91 & 1.25 \\
Quebec & 1.08 & 1.22 & 1.50 \\
Saskatchewan & 0.71 & 0.88 & 0.93 \\
\hline
\end{tabular}

The relative GERD indicators for the European innovation system ranged from about 0.20 to 2.5. In comparison the relative GERD indicators for the Canadian systems ranged from 0.60 to 1.5. An analysis of the variances from the population mean was performed assuming that the nations and provinces listed in the tables represent the entire European and Canadian innovation systems. This assumption is not quite true because Luxembourg and two Canadian territories have not been included due to lack of GERD data. Also, Statistics Canada reports the Federal funding for the Nation Capital 
Region ${ }^{14}$ (NCR) separate from the provincial funding. The NCR values accounted for approximately $3 \%$ of the total GERD in 2000 and they have not included.

The variance from the population mean of the relative GERDs for each innovation system was calculated for each year and the values are plotted in Figure 4. It can be seen that the variance for the European innovation system decreased from above 0.40 to about 0.29 in the first half of the time period and levelled off for the second part of the time period. On the other hand the variance from the population mean for the Canadian innovation system was about 1/10 as large as the variance for the European innovation system and it varied comparatively little over the time period.

\section{[Figure 4 here]}

The larger variances of the relative GERD in the European innovation system compared to the Canadian innovation system can be partially explained by time span over which the innovation systems have been evolving and the differences in their governance structures. Most Canadian provinces have been in the Canadian confederation for over 100 years but some European nations have only been in the European Union for a couple of decades. Also, Europe is a union based on treaties. Canada is a confederation with a central federal government. The governance of European innovation system is more decentralized than the Canadian system. It seems natural to assume that the characters of the two innovation systems will be different. For example, the European Union has less influence on national R\&D expenditures than the Federal government has over the R\&D expenditures by the provinces. Less variance from the systemic scaling trend could be indicative of a system whose members are more tightly integrated. It seems that the time dependent variance from the systemic scaling trend can be used as an indicator of systemic integration.

\section{A Scale-independent Model of an Innovation System}

The scaling relationships between GERD and GDP over time and at points in time can be combined to build a composite scale-independent model that illuminates how they

\footnotetext{
${ }^{14}$ Canada's National Capital region is centred upon the cities of Ottawa in Ontario and Gatineau in Quebec.
} 
emerged together. Figure 5a and 5b contains log-log plots of GERD versus GDP for the European and Canadian innovation systems. The circles are the 1990 data points seen in Figures $3 a$ and 3b, respectively. The dotted lines are the 1990 regression lines seen in the same figures. The long solid dark lines are power law regressions at two other points in time across the national and provincial systems of innovation in 1981 (lower) and 2000 (upper). The systemic scaling factors and $\mathrm{R}^{2}$ values for the three regression lines are given in the top left hand corner of the figures. The short light lines are power regression lines representing the scaling correlation between the exponential growth rates between GERD and GDP for each nation and province. The scaling factors are the slopes of the power law regression lines and they were given in Table 1. For example, in Figure 5a the short line labelled UK for the United Kingdom gives the scaling correlation between the exponential growth rates of the GERD and GDP from 1981 to 2000 . The scaling factor was 0.73 indicating that the UK GERD did not grow as fast as its GDP. On the other hand the short line labelled FIN for Finland had a scaling factor of 2.05. Its GERD grew much faster than its GDP.

\section{[Figure 5 here]}

Figure 6 is a plot of the values of the systemic GERD-GDP scaling factors for the European and Canadian systems over the time period. The system scaling factor for the European innovation system had an obvious decline from $1.31 \pm 0.19$ in 1981 to $1.02 \pm$ 0.15 in 2000 . The Canadian systemic scaling factor was $1.09 \pm 0.06$ in 1981 and $1.14 \pm$ 0.07 in 2000.

\section{[Figure 6 here]}

Table 1 and Figure 5a give clues as to why the European systemic scaling factor decreased with time. The GERDs of the small and medium sized nations tended to grow significantly faster than their respective GDPs. In contrast the GERD of the larger nations like Italy, UK, France and Germany grew close to the same as or slower than their respective GDPs. The systemic tendency of the European innovation system was for the small and medium sized members to force the lower GDP end of the systemic scaling correlation up with time and the larger nations tended to move the upper end down or at least maintain a level close to status quo. Overall, the systemic GERD-GDP 
scaling factor for the European innovation system changed from being quite nonlinear (1.31) to being more linear (1.02). If the trend continues it will become nonlinear again as GERD will be growing at a slower rate than GDP. In comparison the GERDs of every Canadian province grew close to or faster than their respective GDPs. The larger provinces, particularly Quebec, grew their GERDs at rates similar in magnitude to the rates of the small and medium sized European nations. It is unclear if the tendency of the systemic GERD-GDP scaling factor for the Canada innovation system is increasing or perhaps fluctuating around 1.1 or there about. This issue will be explored in the next section.

\section{Using a Scale-independent Model}

It was demonstrated in the previous sections that scaling correlations exist between GERD and GDP across European nations and Canadian provinces at points in time. Also, it was shown that the value of the systemic scaling factor can change over time. The systemic scaling factors are not mathematically predictable from the underlying exponential growth rates; however, they can be measured. The exponential growth trends can be used to predict future values of GERD and GDP and then these values can be used to measure the systemic scaling factor at a point in the future.

Consider the following. If GERD and GDP had exhibited perfect exponential growth then their future values would be exactly predictable and the future values of the systemic scaling factor could be accurately measured. In fact, if the exponential growth was prefect then all past and future values could be predicted from any two consecutive years of data. However, GERD and GDP do not exhibit exactly exponential growth rates; they only exhibit a tendency to grow exponentially. It takes more than two consecutive years of data to identify the trend. The longer the time window over which the observations are made the more accurately the trend can be predicted. For example, the scale-independent models in the previous section were constructed using a 20 year time window. They could have been built using a different size of time-window. A smaller observation window would capture more recent trends but at the cost of losing longer term accuracy in the model.

Scale-independent models were constructed using 20 and 5 year observation time windows and then they were used to predict the size of national and provincial GERDs 
and GDPs in the year 2005. Table 5 and Table 6 give the scaling factor for the power law relationship between GERD and GDP measured using the 5 year observation window from 1996 to 2000 . As expected the $R^{2}$ values for these scaling factors tended to be lower that those seen in Table 1 and Table 2 where a 20 year observation window was used.

Table 5 - European Innovation System (1996-2000)

\begin{tabular}{lccc}
\hline Country & $\mathbf{\alpha}$ & $\mathbf{s e}^{*}$ & $\mathbf{R}^{\mathbf{2}}$ \\
\hline Austria & 2.06 & \pm 0.20 & 0.97 \\
Belgium & 1.70 & \pm 0.05 & 1.00 \\
Denmark & 1.94 & \pm 0.15 & 0.98 \\
Finland & 2.16 & \pm 0.20 & 0.97 \\
France & 0.74 & \pm 0.11 & 0.94 \\
Germany & 1.84 & \pm 0.13 & 0.98 \\
Greece & 2.38 & \pm 0.44 & 0.91 \\
Ireland & 0.66 & \pm 0.04 & 0.99 \\
Italy & 1.28 & \pm 0.16 & 0.96 \\
Netherlands & 0.75 & \pm 0.15 & 0.89 \\
Portugal & 1.92 & \pm 0.24 & 0.95 \\
Spain & 1.53 & \pm 0.13 & 0.98 \\
Sweden & 1.38 & \pm 0.19 & 0.95 \\
United Kingdom & 0.98 & \pm 0.14 & 0.94 \\
\hline * & & &
\end{tabular}

${ }^{*}$ se is the standard error for $\alpha$

Table 6 - Canadian Innovation System (1996-2000)

\begin{tabular}{lccc}
\hline Province & $\boldsymbol{\alpha}$ & $\mathbf{s e}^{{ }^{*}}$ & $\mathbf{R}^{\mathbf{2}}$ \\
\hline Alberta & 0.58 & \pm 0.19 & 0.76 \\
British Columbia & 2.52 & \pm 0.31 & 0.96 \\
Manitoba & 2.34 & \pm 0.61 & 0.83 \\
New Brunswick & 0.70 & \pm 0.58 & 0.33 \\
Newfoundland Labrador & 1.00 & \pm 0.18 & 0.91 \\
Nova Scotia & 1.74 & \pm 0.28 & 0.93 \\
Ontario & 1.55 & \pm 0.14 & 0.97 \\
Prince Edward Island & 4.24 & \pm 0.36 & 0.98 \\
Quebec & 1.53 & \pm 0.12 & 0.98 \\
Saskatchewan & 2.45 & \pm 0.80 & 0.76 \\
\hline
\end{tabular}

${ }^{*}$ se is the standard error for $\alpha$

The two models were used to predict the 2005 GERD and GDP values which were then used to calculate the 2005 systemic scaling factors. The European systemic scaling factor was predicted to be $0.93 \pm 0.12$ using a 20 year observation window and $0.92 \pm$ 0.15 using a 5 year observation window. The values of the system scaling factor for the 
Canadian innovation system were projected to be $1.22 \pm 0.06$ and $1.08 \pm 0.09$, respectively. These findings suggest that the systemic scaling factor for the European innovation system will tend to decrease and in fact the GERD is anticipated to grow slower than GDP. However, it is still not clear what will happen to the systemic scaling factor for the Canadian innovation system other than it will likely stay well above 1.0.

\section{Another Scale-independent View}

Population is an important measure of the size of an economic system. It is used to calculate such things as GDP per capita, an indicator that is frequently used to compare the income of nations. We know from the preceding discussion that the growth of GDP can be approximated by an exponential growth trend. An examination of the growth trends of the European and Canadian populations showed that they tended to growth exponentially too. Over the 20 year time interval the European population tended to grow by $0.31 \%$ per annum and the Canadian population grew by $1.16 \%$ per annum.

Figure 7 is a log-log plot of GDP versus population for Europe and Canada. A predictable scaling correlation exists between these two measures. The predicted value of the scaling factor for the European innovation system was $0.051 \pm 0.001 / 0.003 \pm$ $0.000=16.30$. The measured value was 15.96. The predicted value of the scaling factor for the Canadian innovation system was $0.053 \pm 0.002 / 0.012 \pm 0.000=4.56$. The measured value was $4.54 \pm 0.18$. In both systems the actual data exhibit similar patterns of distribution about the predicted scaling trend lines. This pattern might be indicative of other underlying trends in such things as migration and economic factors. This requires further investigation. However, it can be said with confidence that the scaling relationship between GDP and population in Europe and Canada observed from 1981 to 2000 is reasonably predictable. Also, it tells us that a doubling of the population would be expected to increase GDP by nearly 638,000 times $\left(2^{15.96}\right)$ in Europe but only $23.6\left(2^{4.56}\right)$ times in Canada. The large difference in the scaling factors can be explained by the fact that the $\mathrm{GDP}_{\mathrm{C}}-\mathrm{GDP}_{\mathrm{E}}$ scaling factor was 1.03 (see section 4.1) and the $\mathrm{POP}_{\mathrm{C}}-\mathrm{POP}_{\mathrm{E}}$ scaling factor was measured to be 3.69. This indicates that while the European and Canadian GDPs are growing at similar rates the Canada's population was growing nearly 4 times as fast as the European population. It will take Europe a much longer to double its size that it will take Canada. The GDP-POP scaling factors also indicate that 
GDP per capita would expected to increase $32,000\left(2^{14.96}\right)$ and $12\left(2^{3.56}\right)$ fold, respectively, each time the GDP doubles.

[Figure 7 here]

Figures 8 and 9 and Tables 7 and 8 give the highlights of scale-independent models for Europe and Canada based on the exponential growth of population and GDP between 1981 and 2000. Figure 8a contains a variety of log-log plots of GDP versus population for Europe. As in Figure 5a the circles are the 1990 data points and there are three power law regression lines. The dotted line is the regression line through the 1990 data. The lower line is the regression line through the 1981 data and the upper line is through the 2000 data. The scaling factors of the regression lines are given in the upper right hand corner of the graph. The shorter lines give the scaling correlation between GDP and population for each European country. Figure $8 \mathrm{~b}$ contains a similar plot for Canada. Figure 9 is a plot of the value of the systemic scaling factor over time.

[Figure 8 here]

\begin{tabular}{lrcc}
\multicolumn{4}{c}{ Table 7 - European GDP-Population Scaling Factors } \\
\hline \multicolumn{1}{c}{ Country } & A & se $^{*}$ & $\mathbf{R}^{\mathbf{2}}$ \\
\hline Austria & 11.86 & \pm 0.97 & 0.89 \\
Belgium & 19.01 & \pm 1.20 & 0.93 \\
Denmark & 17.39 & \pm 1.81 & 0.84 \\
Finland & 11.18 & \pm 0.67 & 0.94 \\
France & 10.42 & \pm 0.13 & 1.00 \\
Germany & 12.51 & \pm 1.19 & 0.86 \\
Greece & 8.09 & \pm 0.20 & 0.99 \\
Ireland & 16.70 & \pm 1.75 & 0.84 \\
Italy & 33.59 & \pm 3.80 & 0.81 \\
Netherlands & 8.95 & \pm 0.21 & 0.99 \\
Portugal & 25.15 & \pm 6.47 & 0.46 \\
Spain & 22.55 & \pm 0.87 & 0.97 \\
Sweden & 10.06 & \pm 0.77 & 0.90 \\
United Kingdom & 23.21 & \pm 1.00 & 0.97 \\
\hline * se is the standard error for $\alpha$ & &
\end{tabular}




\section{Table 8 - Canadian GDP-Population scaling factors}

\begin{tabular}{lrll}
\hline Province & $\boldsymbol{\alpha}$ & $\mathbf{s e}^{\boldsymbol{*}}$ & $\mathbf{R}^{\mathbf{2}}$ \\
\hline Alberta & 3.37 & \pm 0.16 & 0.96 \\
British Columbia & 2.77 & \pm 0.12 & 0.97 \\
Manitoba & 9.20 & \pm 0.30 & 0.98 \\
New Brunswick & 14.78 & \pm 0.89 & 0.94 \\
Newfoundland and Labrador & -6.01 & \pm 1.58 & 0.44 \\
Nova Scotia & 10.38 & \pm 0.43 & 0.97 \\
Ontario & 3.81 & \pm 0.18 & 0.96 \\
Prince Edward Island & 10.71 & \pm 0.60 & 0.95 \\
Quebec & 7.27 & \pm 0.38 & 0.95 \\
Saskatchewan & 5.12 & \pm 4.17 & 0.08 \\
\hline
\end{tabular}

${ }^{*}$ se is the standard error for $\alpha$

Tables 7 and 8 show that the reliability of the GDP-Population scaling factors for some smaller nations and provinces are questionable. For example the GDP-Population scaling factor for Saskatchewan has a large standard error and a low $\mathrm{R}^{2}$ value. This occurred because while the provincial GDP exhibited exponential growth the population had both positive and negative growth periods. Also, the scaling factors in Europe tended to be larger and had less variation in their ranges than those for Canada. This is illustrated by the fact that the scaling factors in Europe ranged from $8.09 \pm 0.20$ for Greece to $33.40 \pm 3.80$ for Italy and had an average magnitude of 16.5. In Canada they ranged from $-6.01 \pm 1.58$ for NL to $14.8 \pm 0.08$ for New Brunswick with an average magnitude of 6.4 .

\section{[Figure 9 here]}

Figure 9 suggests that the systemic GDP-Population scaling factor is decreasing for Europe and Canada. It was quite constant in Europe staying around 1.02 to 1.04 until the mid 1990s and then declined dipping below 1.0 in 1998. Over the same interval the systemic GDP-population scaling factor for Canada decreased from 1.16 and then appeared to level off around 1.1.

Figure $8 \mathrm{~b}$ illustrates an interesting point. A scale-independent model can accommodate exponential decreases. Newfoundland and Labrador (NL) exhibited a decline in population and GDP over the 20 year time frame as seen by the negative slope of its power law regression line. Also, a scale-independent model can accommodate the case where one variable exhibits exponential growth and the other exponential decay. 


\section{Summary and Conclusions}

In summary, scaling correlations between GERD \& GDP and GDP \& population have been shown for the European and Canadian innovation systems. The scaling correlations that emerge within the national and provincial innovation systems are predictable from the underlying exponential growths of GERD, GDP and population. The scaling correlations that exist across national and provincial innovation systems at points in time are not mathematically predictable but they are measurable. In other words, in the examples the scaling correlations over time appeared to be more deterministic than the scaling correlations at points in time.

The European and Canadian innovation systems exhibit emergent properties. For example, the systemic scaling relationship between GERD \& GDP and GDP \& population at points in time are not predictable and they are not solely determined by any national or provincial innovation system. A systemic scaling relationship is determined by the complex activities of the member systems within the European and Canadian innovation systems. Furthermore, the systemic scaling factor can change with time indicative of another emergent property.

Scale-independent indicators were constructed statistical measures commonly used to construct conventional indicators of innovation systems. Unlike conventional indicators scale-independent indicators can be used to compare systems of different sizes. For example, the GERD-GDP scaling factors can be used to compare the relative growth rates of GERD and GDP of members in the same and in different innovation systems. Similarly, the system GERD-GDP scaling factor can be used to compare how the GERDs in an innovation system tends to increase with increasing GDPs at points in time.

R\&D intensity, GDP per capita and citations per paper ${ }^{15}$ are indicators commonly used to compare innovation systems. They are used by governments and agencies to measure performance, set targets and inform public policy. It has been demonstrated that the measures used to derive the ratios in these cherished conventional indicators tend to scale with size (GDP, population and papers). They should not be used to compare

${ }^{15}$ Scaling correlations between citations and papers have been shown in my previous papers (Katz, 2005). 
innovation systems of different sizes unless they have been adjusted for scaling tendencies. Scale-independent indicators are more robust than conventional indicators because they can account for systemic behaviours in an innovation system. These indicators will assist innovation system observers to compare small and large players in a complex innovation system without the non-linear distortion introduced through the use of conventional indicators.

Scale-independent indicators can be used to construct scale-independent models of innovation systems. These models provide insights into how the systemic scaling relationships emerged with time due to changes in the internal dynamics of the system. In turn the variances of member systems about a systemic scaling correlation trend can provide insights into how a collection of regional or national innovation systems are evolving into national and supranational innovation systems. In other words, information derived from scale independent models can have policy relevance relating to the cohesion and integration of an innovation system. Also by using different sized time windows of observation the model can be used to calculate how the system scaling factor is emerging in the shorter and longer term.

Innovation systems are complex systems with many interacting complex subsystems. They can exhibit predictable and measurable scaling characteristics. The scaling correlations can be used to build scale-independent indicators which in turn can be used to construct scale-independent models. The evolution of the scaling correlations can be used to identify emergent properties of an innovation system and in turn inform public policy.

\section{Acknowledgements}

The author would like to thank Viv Cothey, Ben Martin, Benoit Godin, Frank Havemann, Diana Hicks, Cooper Langford and Roland Wagner-Döbler for their insightful comments and suggestions. Finally the author would like to thank his father, Leon Katz, for the many years of discussion and assistance in developing the theory of scale-independent indicators. He is lovingly missed. 


\section{References}

Adams, D. (2002). The counting house. Nature, 415, 726-729 http://www.nature.com/nature/journal/v415/n6873/pdf/415726.pdf.

Albert, R., \& Barabasi, A.-L. (2002). Statistical mechanics of complex networks. Reviews of Modern Physics, 74 http://www.nd.edu/ networks/PDF/rmp.pdf.

Amaral, L. A. N., Buldyrev, S. V., Havlin, S., Salinger, M. A., \& Stanley, H. E. (1998). Power Law Scaling for a System of Interacting Units with Complex Internal Structure. Physical Review Letters, 80(7) http://polymer.bu.edu/hes/articles/abhss98.pdf.

Amaral, L. A. N., Gopikrishnan, P., Plerou, V., \& Stanley, H. E. (2001). A model for the growth dynamics of economic organizations. Physica A, 299, 127-136 http://amaral.chem-eng.northwestern.edu/Publications/Papers/Amaral-2001PhysicaA-299-127.pdf.

Amaral, L. A. N., \& Ottino, J. M. (2004). Complex networks: Augmenting the framework for the study of complex systems. Eur. Phys. J. B, 38, 147-162 http://amaral.chem-eng.northwestern.edu/Publications/Papers/Amaral-2004Eur.Phys.J.B-38-147.pdf.

Barabasi, A.-L., \& Albert, R. (1999). Emergence of Scaling in Random Networks. Science, 286(October 15), 509-512 http://www.nd.edu/ networks/Papers/science.pdf.

Barabasi, A.-L., Ravasza, E., \& Vicsek, T. (2001). Deterministic scale-free networks. Physica A, 299, 559-564 http://www.nd.edu/ networks/PDF/Deterministic.pdf.

Barnsley, M. (1988). Fractals Everywhere: Academic Press Inc.

Blok, H. J. (2000). On the Nature of the Stock Market: Simulation and Experiments. Unpublished Doctor of Philosophy, University of British Columbia, Vancouver, $\mathrm{BC}$, Canada.

Carlson, J. M., \& Doyle, J. C. (2002). Complexity and Robustness. PNAS, 99(February 19), 2538-2545 http://www.pnas.org/cgi/reprint/99/suppl 1/2538.pdf.

Christensen, K., Danon, L., Scanlon, T., \& Bak, P. (2002). Unified scaling law for earthquakes. PNAS, 99, 2509-2513 http://www.pnas.org/cgi/reprint/99/suppl 1/2509.

De Solla Price, D. J. (1963). Little Science, Big Science. New York and London: Columbia University Press.

Dorogovtsev, S. N., Goltsev, A. V., \& Mendes, J. F. F. (2001). Pseudofractal Scale-free Web. arXiv:cond-mat/0112143, 1 http://arxiv.org/PS cache/cond-mat/pdf/0112/0112143.pdf.

Faloutsos, M., Faloutsos, P., \& Faloutsos, C. (1999). On Power Law relationships of the Internet Topology. Paper presented at the Sigcomm 1999.

Freeman, C. (1987). Technology and Economic Performance: Lessons from Japan. London and New York: ,Pinter Publishers.

Gibrat, R. (1931). Les Inégalités économiques. Paris, France.

Godin, B. (2005). Measurement and Statistics on Science and Technology: 1920 to the Present: Routledge.

Goldberger, A. L., Amaral, L. A. N., Hausdorff, J. M., Ch. Ivanov, P., Peng, C. K., \& Stanley, H. E. (2002). Fractal dynamics in physiology: Alterations with disease and aging. PNAS, 99, 2466-2247 http://www.pnas.org/cgi/reprint/99/suppl 1/2466. 
Havemann, F., Heinz, M., \& Wagner-Döbler, R. (2005). Firm-like Behaviour of Journals? Scaling Properties of Their Output and Impact Growth Dynamics. Journal of the American Society for Information Science and Technology, 56(1), 3-12 http://141.20.126.8/ fhavem/Havemann-Heinz-WagnerDoeblerJournals Growth Dynamics.pdf.

Holbrook, J. A. D. (1991). The influence of scale effects on international comparisons of R\&D expenditures. Science and Public Policy, 18(4), 259-262

Irvine, J., \& Martin, B. (1983). Assessing Basic Research: The Case of Issac Newton Telescope. Social Studies of Science, 13, 49-86

Katz, J. S. (1999). The Self-Similar Science System. Research Policy, 28, 501-517 http://www.sussex.ac.uk/Users/sylvank/pubs/SSSS.pdf.

Katz, J. S. (2000). Scale Independent Indicators and Research Assessment. Science and Public Policy, 27(1), 23-36 http://www.sussex.ac.uk/Users/sylvank/pubs/SI-RE.pdf.

Katz, J. S. (2005). Scale Independent Bibliometric Indicators. Measurement: Interdisciplinary Research and Perspectives, 3(1), 24-28 http://www.sussex.ac.uk/Users/sylvank/pubs/SIBI.pdf.

Katz, J. S., \& Hicks, D. (1998). Indicators for Systems of Innovation - a bibliometricsbased approach (No. Project No. PL951005 under the Targeted Socio-Economic Research Programme) http://www.sussex.ac.uk/Users/sylvank/pubs/ldea12.pdf.

Katz, J. S., \& Katz, L. (1999). Power Laws and Athletic Performance. Journal of Sports Science, 17, 467-476 http://www.sussex.ac.uk/Users/sylvank/pubs/running.pdf.

Lundvall, B. A. (1992). National Systems of innovation: Towards a theory of innovation and interactive learning. London: Pinter.

Mitzenmacher, M. (2003). A Brief History of Generative Models for Power Law and Lognormal Distributions. Internet Mathematics, 1(2), 226-251 http://www.internetmathematics.org/volumes/1/2/pp226 251.pdf.

Mossa, S., Barthelemy, M., Stanley, H. E., \& Amaral, L. A. N. (2002). Truncation of Power Law Behavior in "Scale-Free" Network Models due to Information Filtering. Physical Review Letters, 88(13) http://amaral.northwestern.edu/Publications/Papers/Mossa-2002-Phys.Rev.Lett.88-138701.pdf.

Neary, J. P. (2005). Purchasing Power Parity. In S. E. J.J. McCusker, L.R. Fischer, D.J. Hancock, K.L. Pomeranz (Ed.), Encyclopedia of World Trade Since 1450. New York: Macmillan Reference.

Newman, M. E. J. (2000). The power of design. Nature, 405

Newman, M. E. J. (2001). The structure of scientific collaboration networks. PNAS, 98, 404-409 http://nicomedia.math.upatras.gr/courses/mnets/mat/Newman StructureOfScienti ficCollaborationNets.pdf.

Newman, M. E. J., Watts, D. J., \& Strogatz, S. H. (2002). Random graph models of social networks. PNAS, 99, 2566-2572 http://www.pnas.org/cgi/reprint/99/suppl 1/2566.

OECD. (1976). Science and Technology Indicators: DSTI/SPR/76.43

Parrish, J. K., Viscid, S. V., \& Grunbaum, D. (2002). Self-Organized Fish Schools: An Examination of Emergent Properties. Biol. Bull., 202, 296-305 http://lims.mech.northwestern.edu/ lynch/IGERT499/parrish-grunbaum-2002.pdf.

Peterson, G. D. (2000). Scaling ecological dynamics: self-organization, hierarchical structure and ecological resilience. Climatic Change, 44, 3 
http://www.geog.mcgill.ca/faculty/peterson/PDF-myfiles/CChange-GDP.pdf.

Plerou, V., Amaral, L. A. N., Gopikrishnan, P., Meyer, M., \& Stanley, H. E. (1999). Similarities between the growth dynamics of university research and of competitive economic activities. Nature, 400, 433-437

Schroeder, M. (1991). Fractals, chaos and power laws. New York: W.H. Freeman and Company.

Schuch, K. (1998). The emergence of the European Innovation System and its impact on the Austrian S\&T system. Paper presented at the Proceedings from the 38th Congress of the European Regional Science Association, Aug - Sept, 28-1, Vienna.

Solomon, S., \& Agay, A. (1997). Power-Laws and Scaling in the Generalized LotkaVolterra (GLV) Model. Paper presented at the 1st Workshop on Econophysics, Budapest.

Stanley, H. E., \& Plerou, V. (2001). Scaling and universality in economics: empirical results and theoretical interpretation. Quantitative Finance, 1, 563-567 http://polymer.bu.edu/hes/articles/qf01.pdf.

StarMap. (2004). A comparative guide to multi actors and multi measures programmes (MAPS) in RTDI policy. http://www.tig.or.at/projekte/euprojekte/DiscoMAP/starMAP.pdf.

Stoneman, P. (1995). Handbook of the Economics of Innovation and Technological Change: Blackwell.

Strogatz, S. H. (2001). Exploring Complex Networks. Nature, 410, 268-276

Strogatz, S. H. (2005). Romanesque networks. Nature, 433(7), 365

Van Raan, A. F. J. (2005). Measurement of central aspects of scientific research: performance, interdisciplinarity, structure. Measurement: Interdisciplinary Research and Perspectives, forthcoming

Van Raan, A. F. J. (2005 (submitted)). Statistical Properties of Bibliometric Indicators: Research Group Indicator Distributions and Correlations. Journal of the American Society for Information Science and Technology http://www.cwts.nl/Cwts/AvRStatProp.pdf.

Voyer, R. (1999). Thirty Years of Canadian Science Policy. Science and Public Policy, 26(4), 277-282

Warhaft , Z. (2002). Turbulence in nature and in the laboratory. PNAS, 99, 2481-2486 http://www.pnas.org/cgi/reprint/99/suppl 1/2481

West, G. B., Woodruff, W. H., \& Brown, J. H. (2002). Allometric scaling of metabolic rate from molecules and mitochondria to cells and mammals. PNAS, 99, 2473-2478 http://www.pnas.org/cgi/reprint/99/suppl 1/2473. 


\section{A. European Innovation System (1981-2000)}

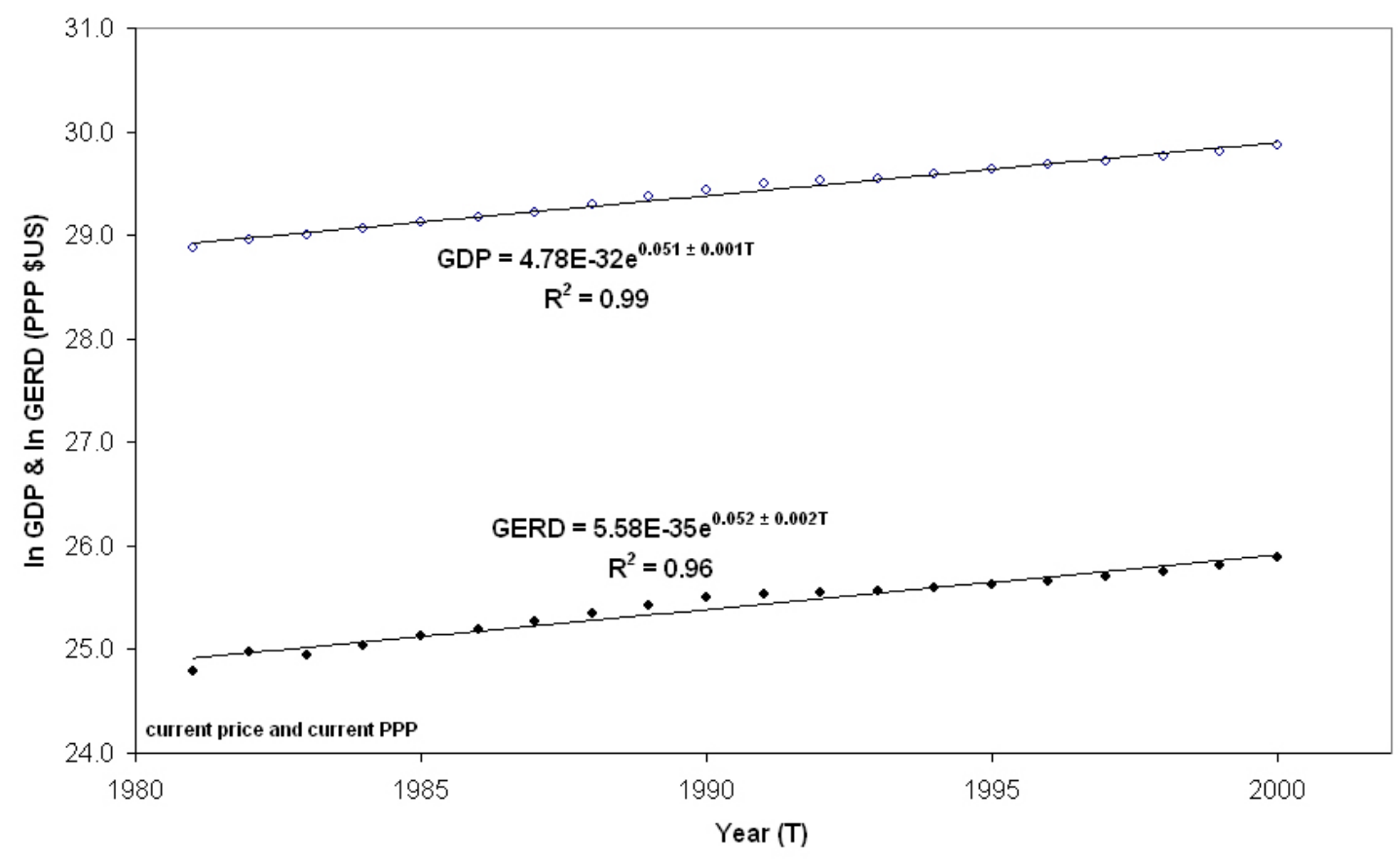

B. Canadian Innovation System (1981-2000)

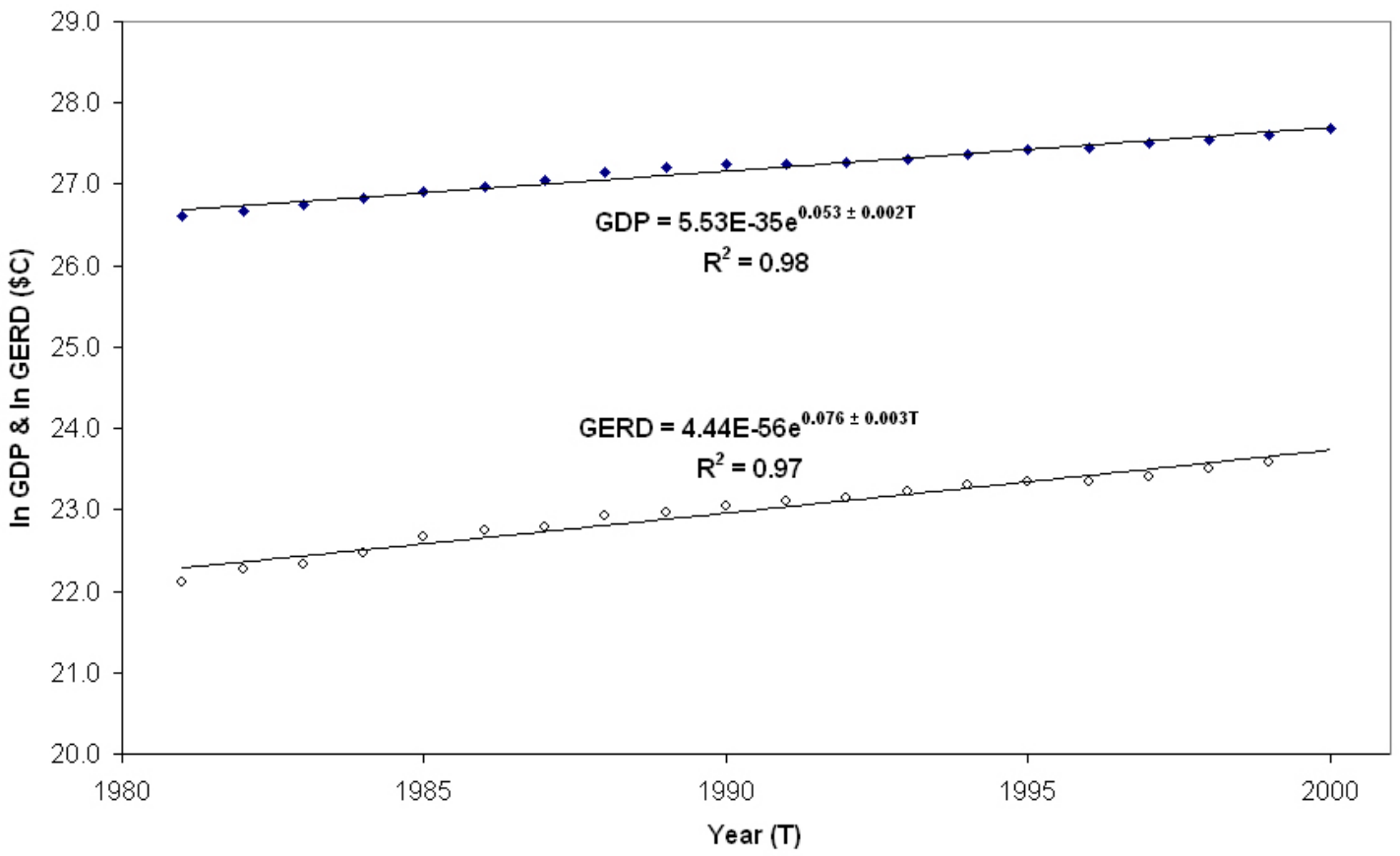

Figure 1 - Growth of GERD and GDP for (A) European Innovation System and (B) Canadian Innovation System from 1981 to 2000. 


\section{A. European Innovation System (1981-2000)}

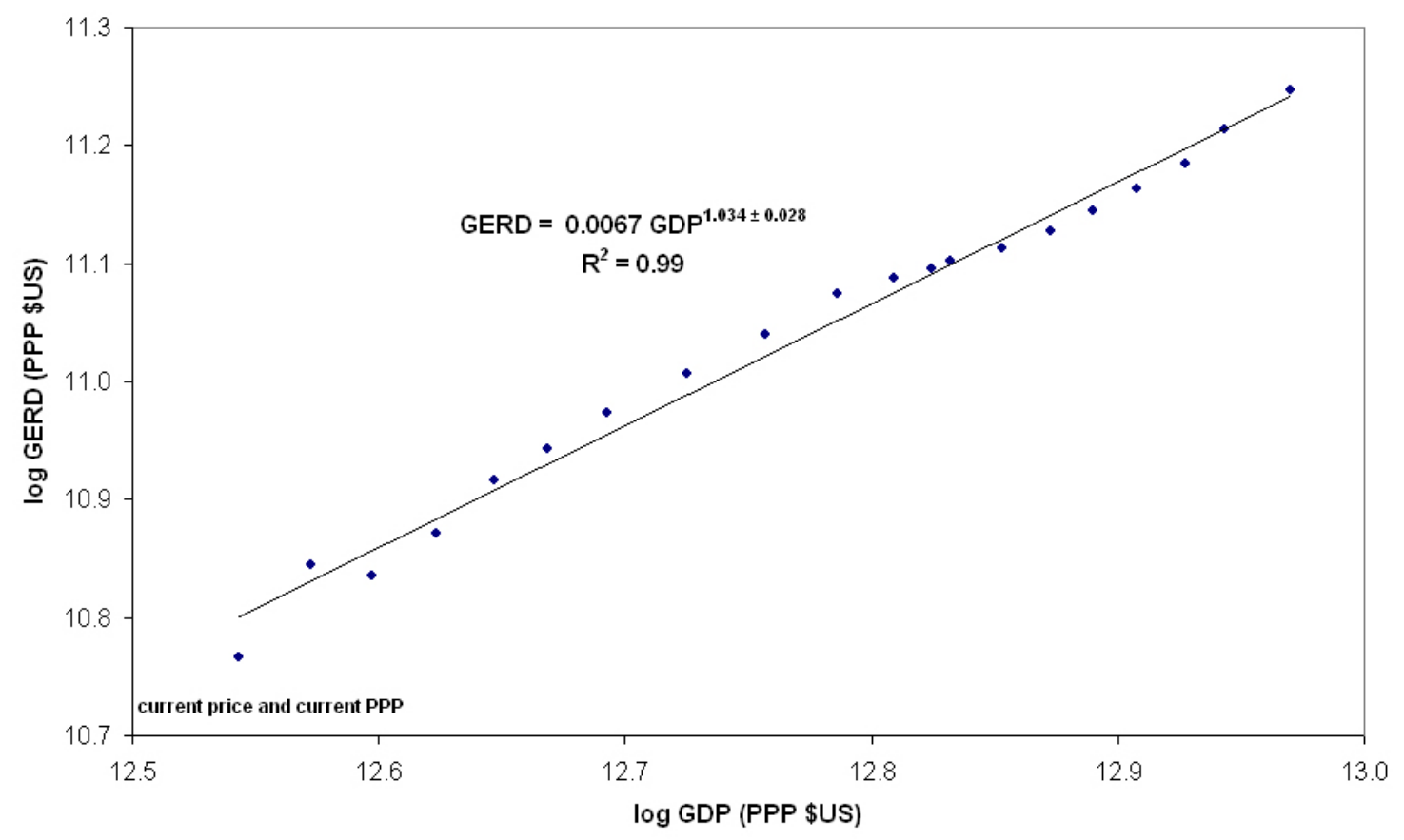

B. Canadian Innovation System (1981-2000)

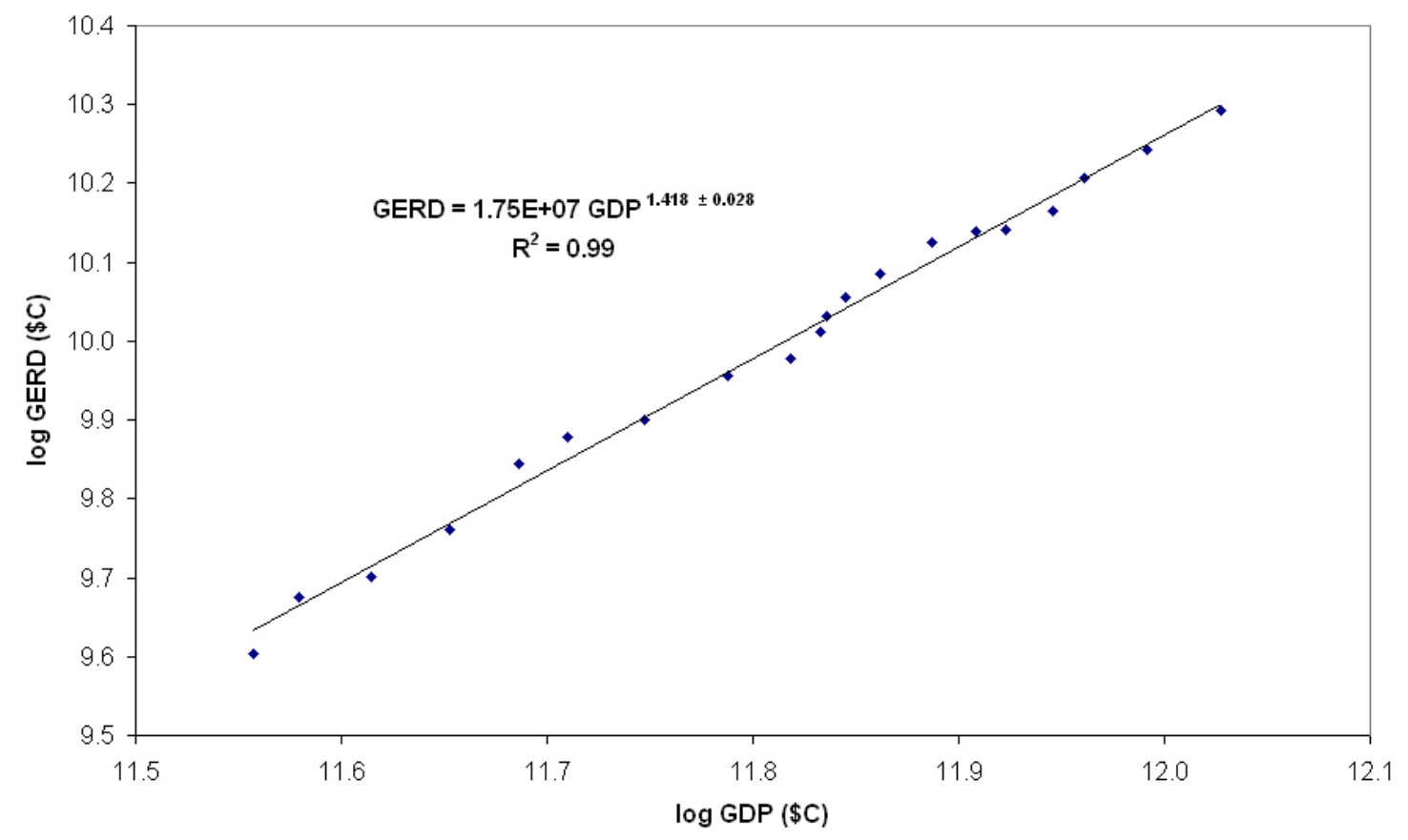

Figure 2 - Scaling correlation between GERD and GDP for (A) European innovation system and (B) Canadian innovation system from 1981 to 2000 


\section{A. European Innovation System (1990)}

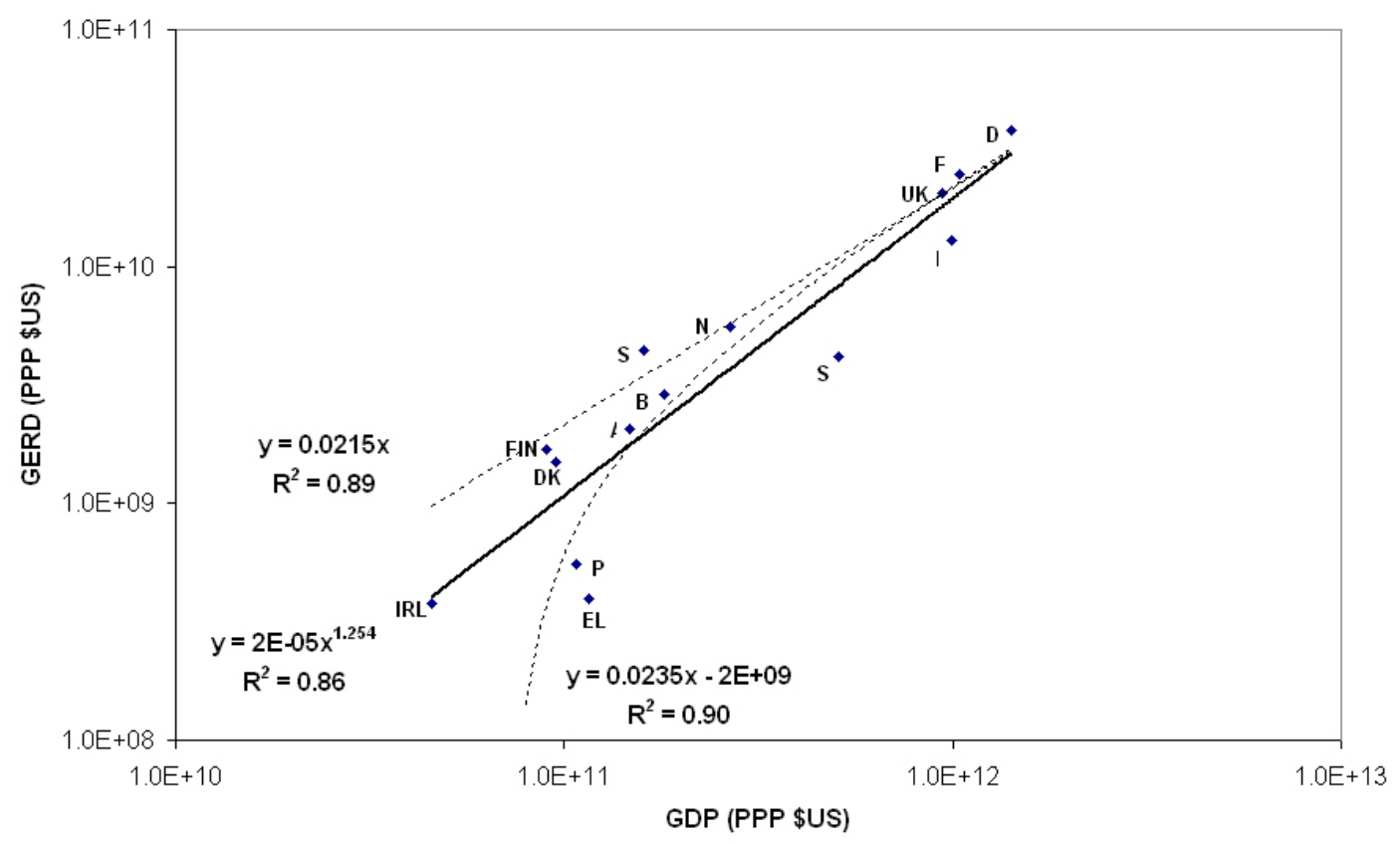

A -Austria, B - Belgium, D - Germany, DK - Denmark, E - Spain, EL - Greece, F - France,

FIN - Finland, I - Italy, IRL - Ireland, NL -Netherlands, P - Portugal, S - Sweden and UK -United Kingdom

\section{B. Canadian Innovation System (1990)}

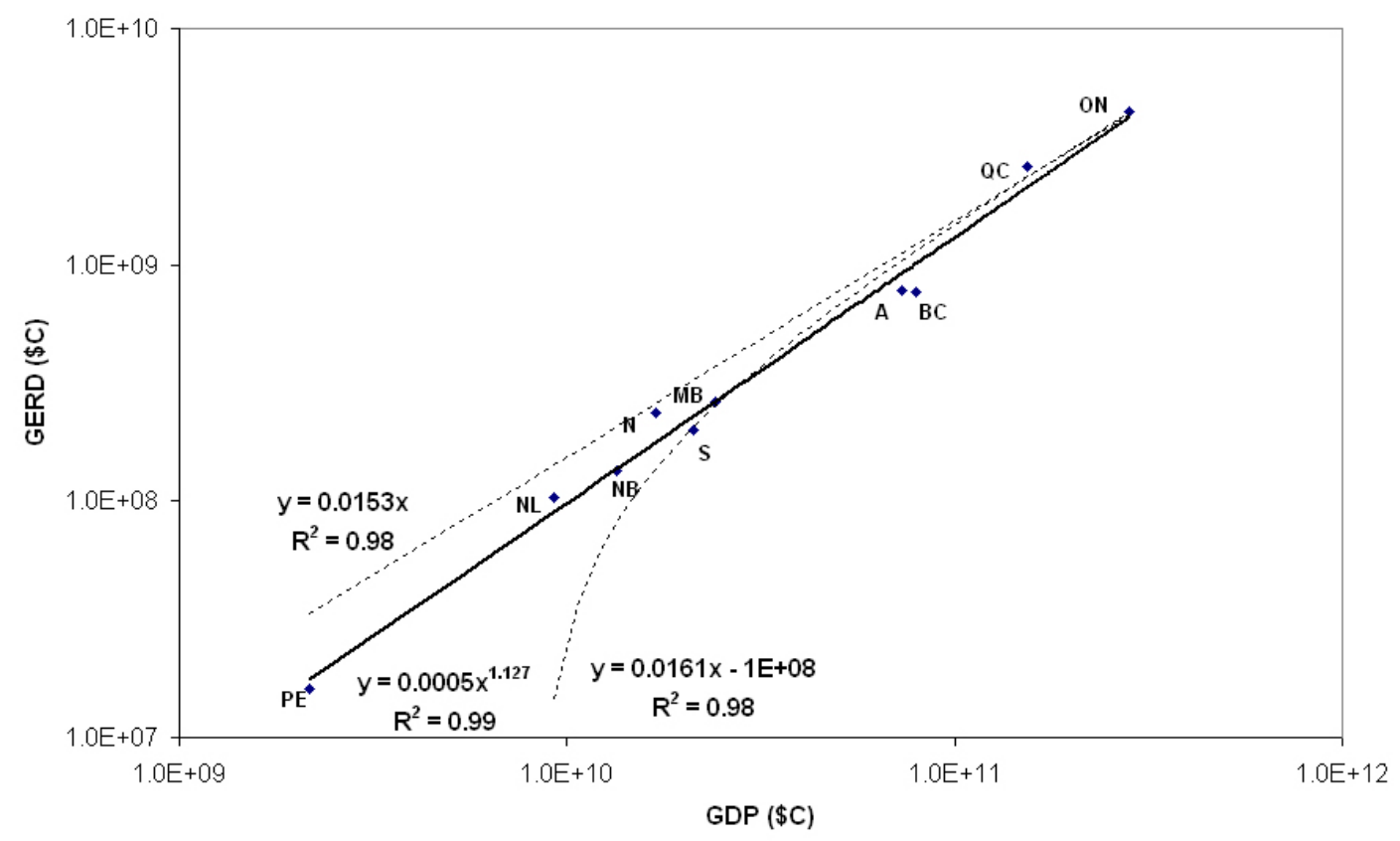

AB - Alberta, BC - British Columbia, MB - Manitoba, NB - New Brunswick, NL -Newfoundland \& Labrador, NS - Nova Scotia, ON - Ontario, PE - Prince Edward Island, QC - Quebec and SK - Saskatchewan

Figure 3 - Systemic scaling correlation between GERD and GDP in 1990 for (A)

European innovation system and (B) Canadian innovation system 


\section{Variance of Relative GERD}

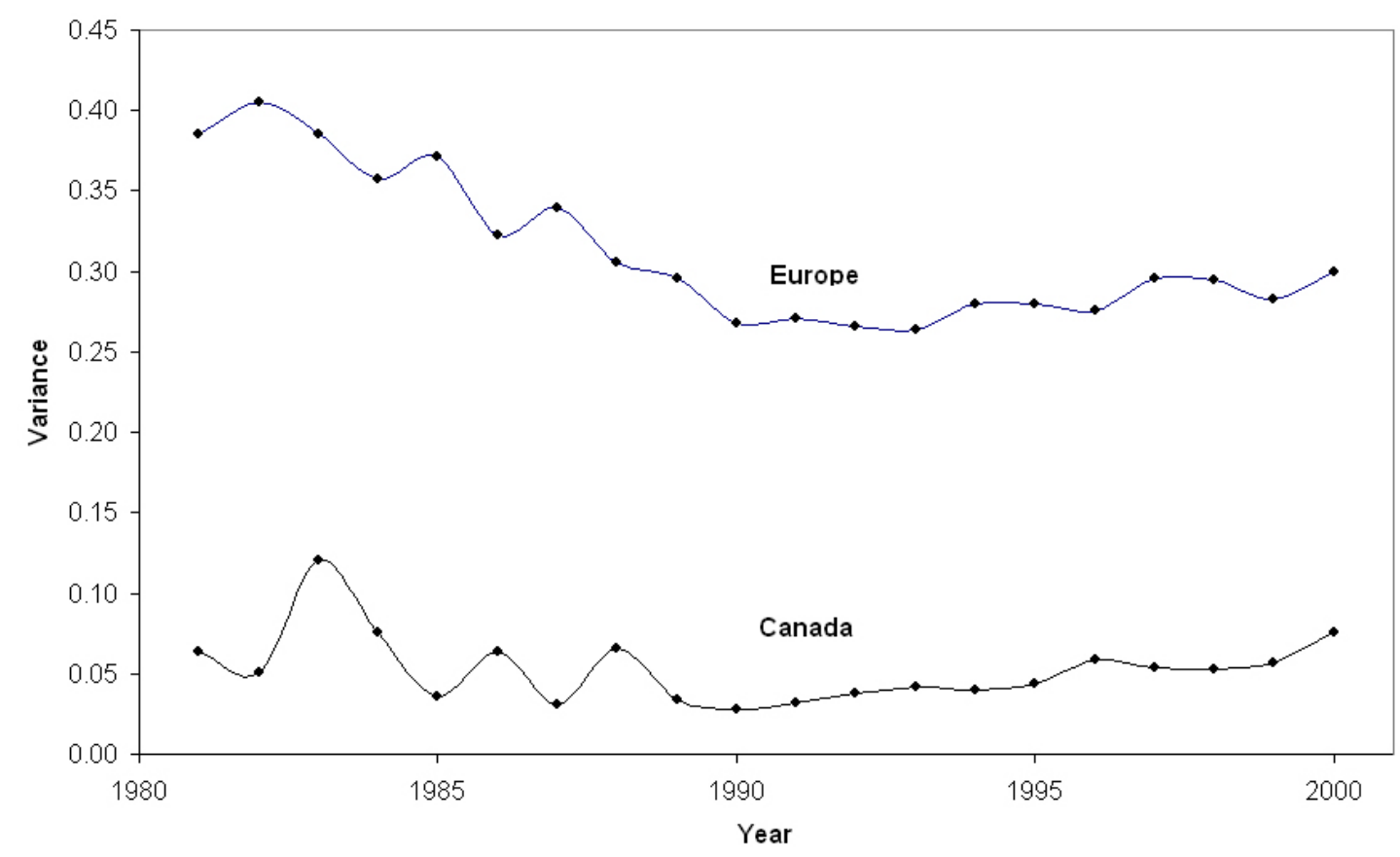

Figure 4 - Variance of the relative GERDs for the European and Canadian innovation systems 


\section{A. Scale Independent Model of the European Innovation System}

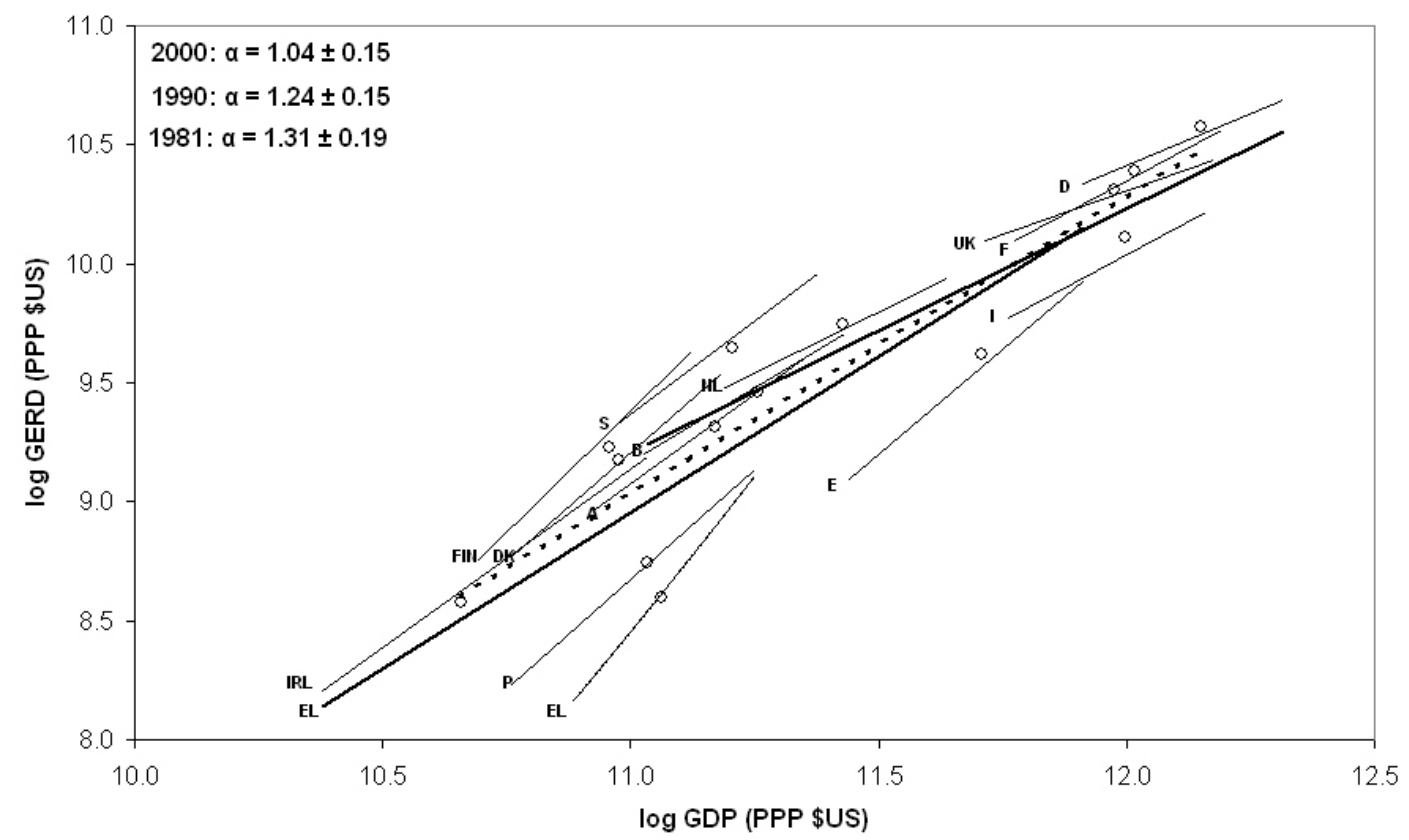

A - Austria, B - Belgium, D - Germany, DK - Denmark, E - Spain, EL - Greece, F - France,

FIN - Finland, I - Italy, IRL -Ireland, NL -Netherlands, P - Portugal, S - Sweden and UK -United Kingdom

\section{B .Scale Independent Model of the Canadian Innovation System}

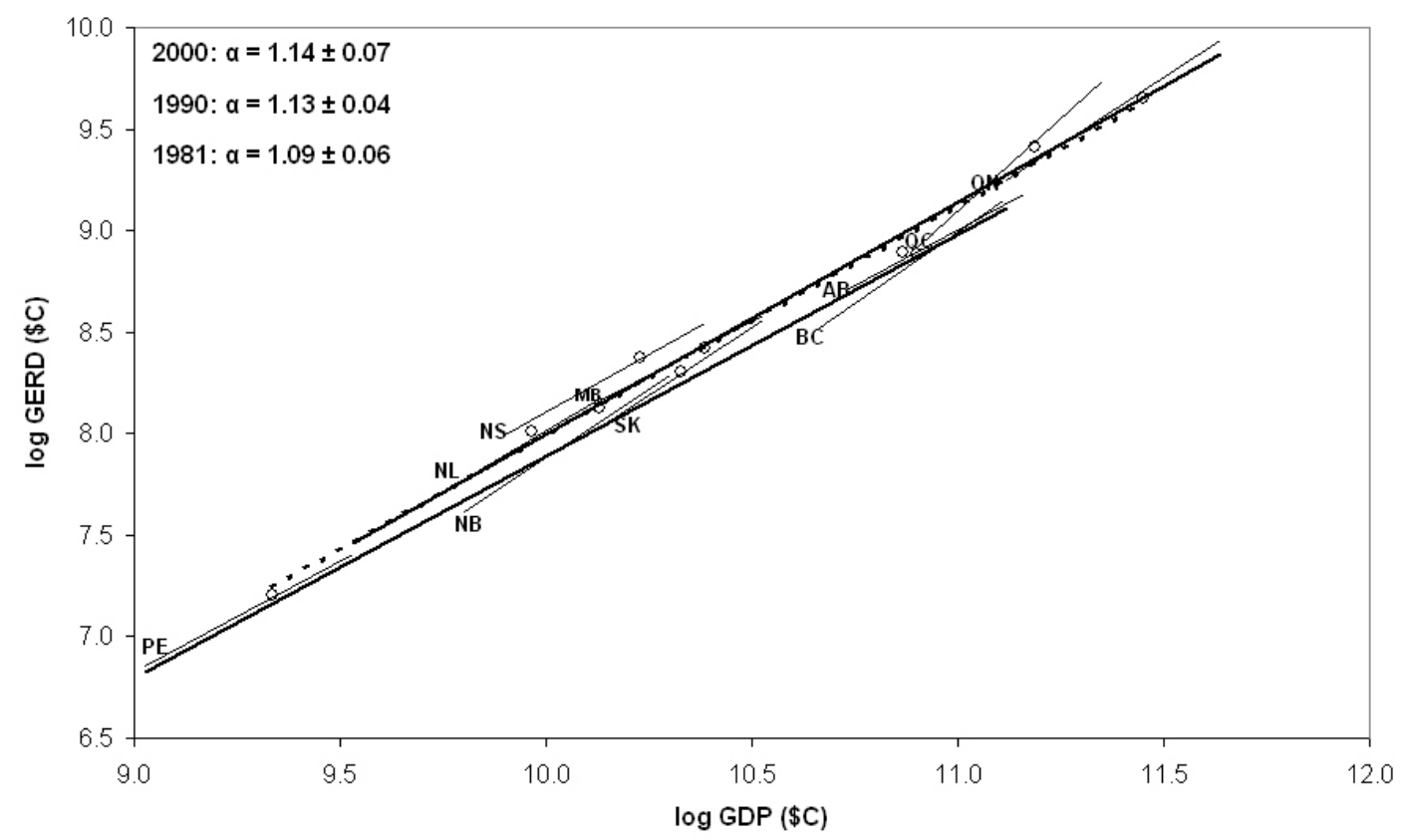

AB - Alberta, BC - British Columbia, MB - Manitoba, NB - New Brunswick, NL -Newfoundland \& Labrador, NS - Nova Scotia, ON - Ontario, PE - Prince Edward Island, QC - Quebec and SK - Saskatchewan

Figure 5 - Scale-independent GERD-GDP models of the (A) European innovation system and (B) Canadian innovation system from 1981 to 2000 


\section{European and Canadian Innovation Systems}

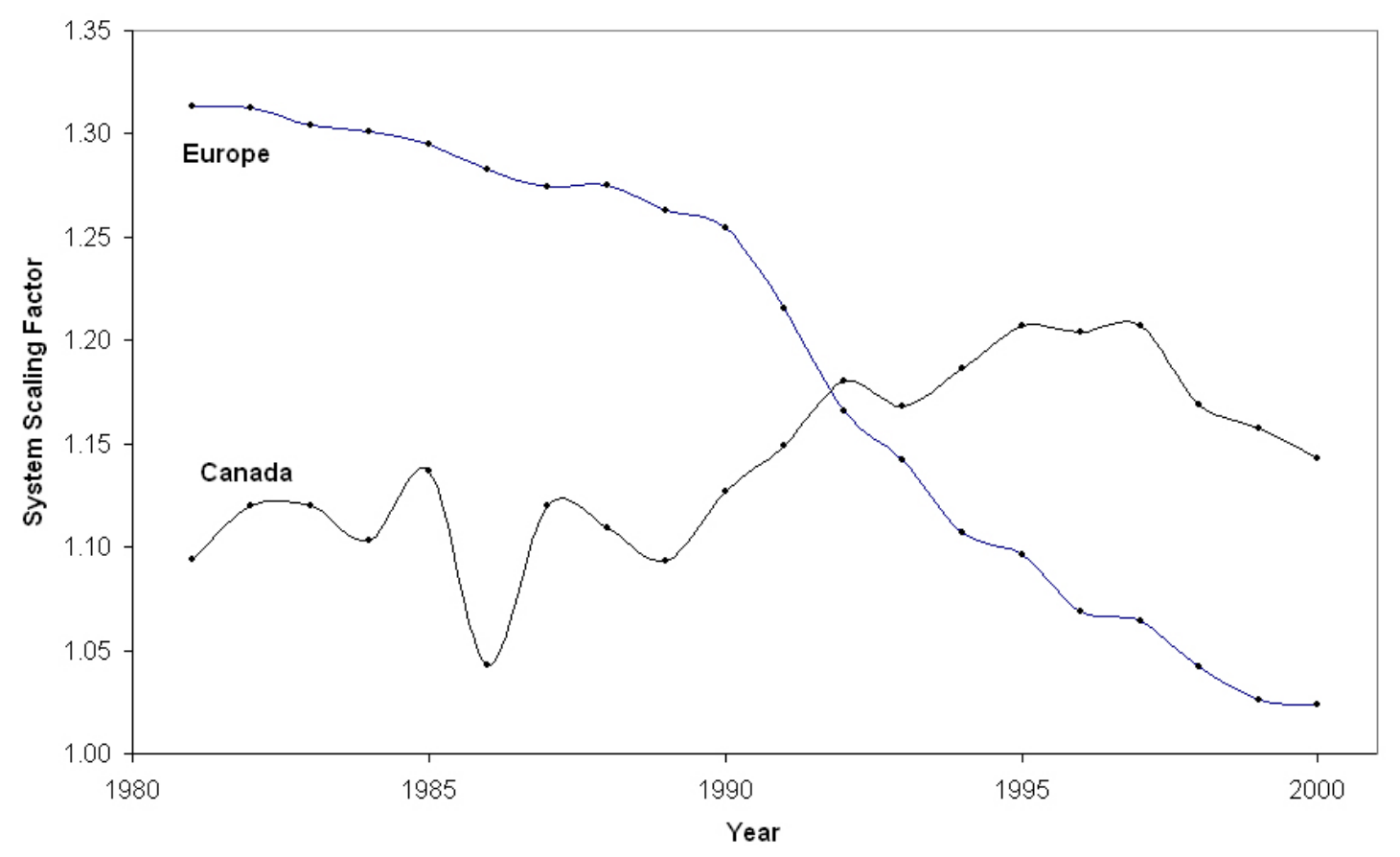

Figure 6 - Value of the GERD-GDP systemic scaling factor over time for European and Canadian Innovation systems 


\section{A. European Innovation System (1981-2000)}

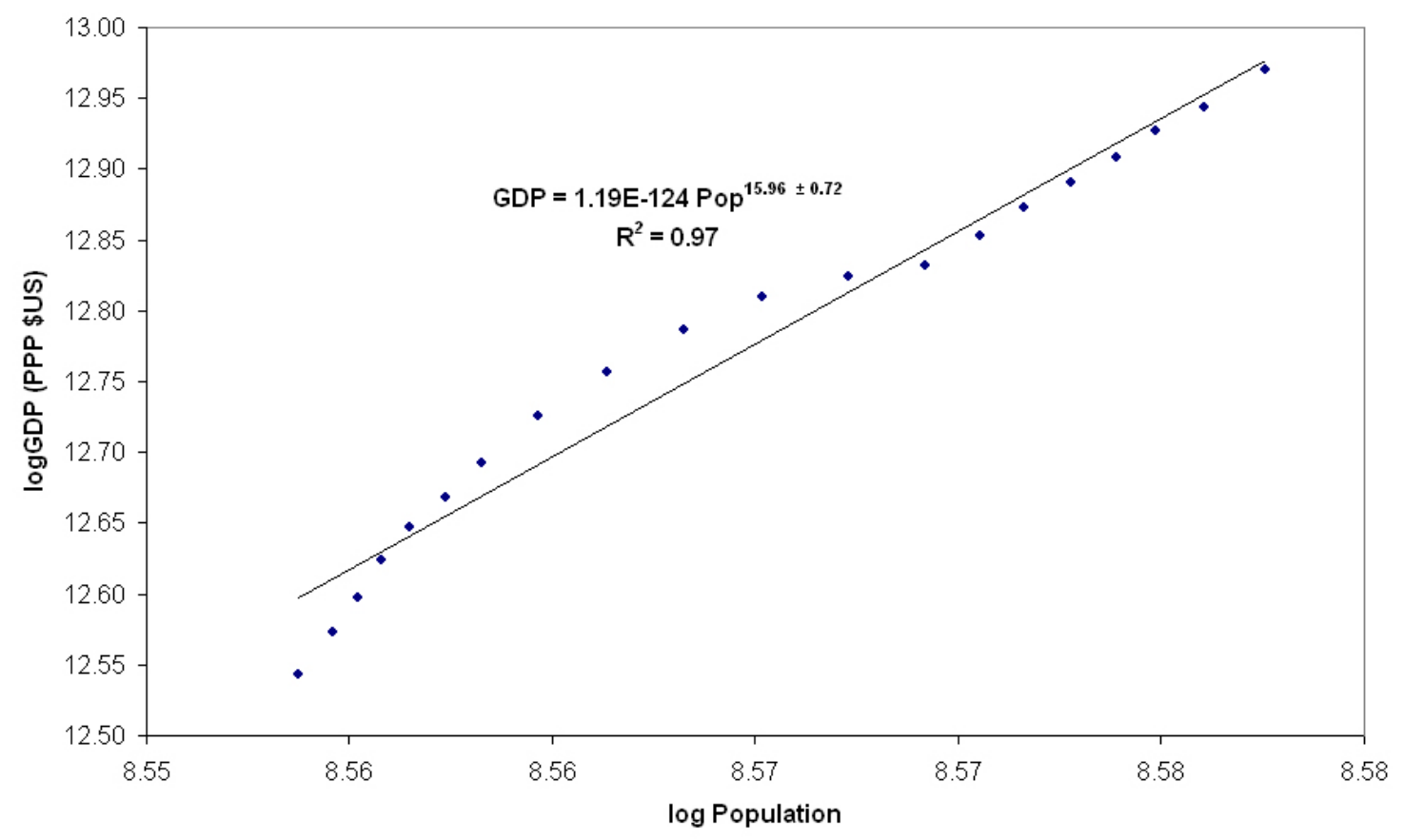

B. Canadian Innovation System (1981-2000)

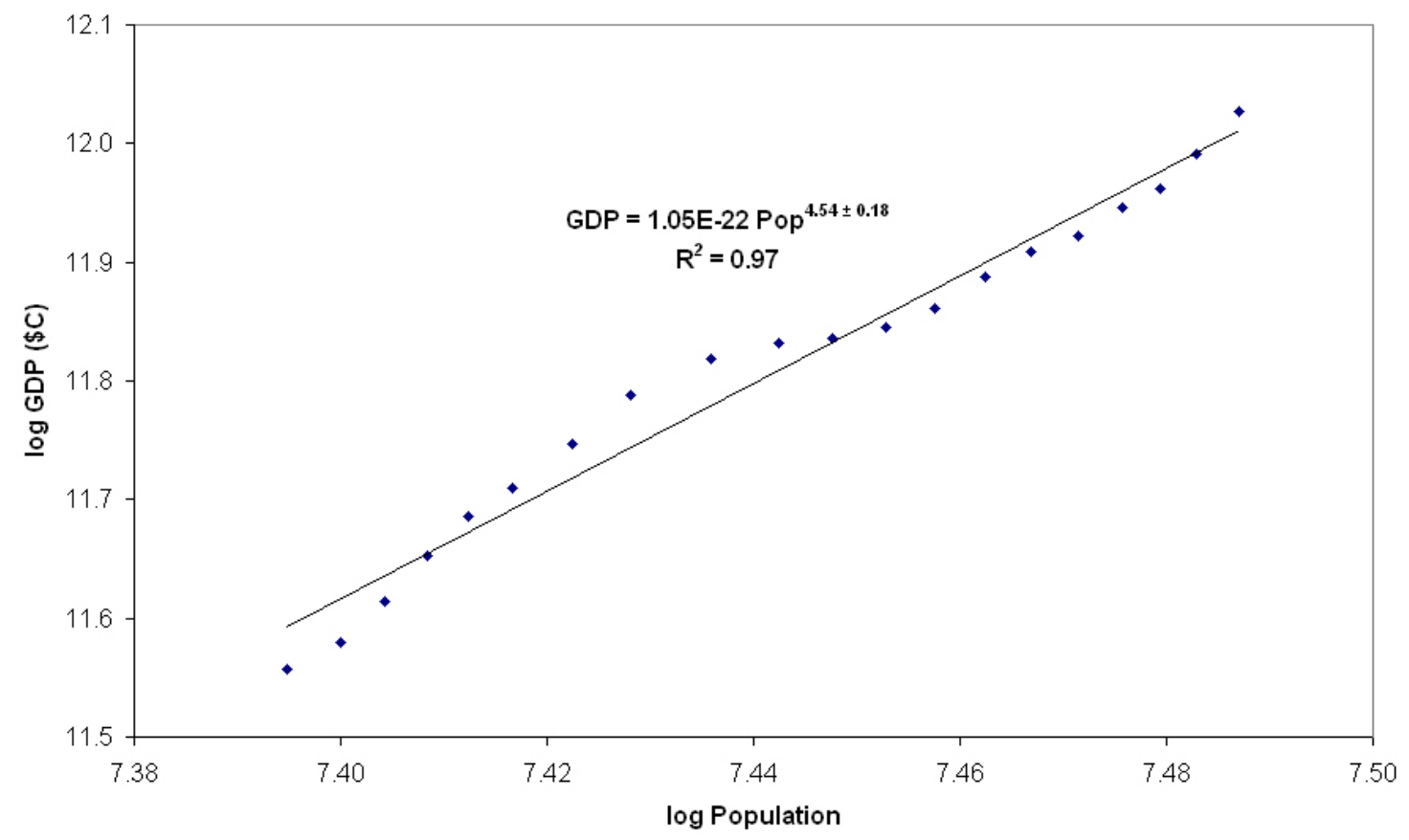

Figure 7 - Scaling correlation between GDP and population for (A) Europe and (B) Canada from 1981 to 2000 


\section{A. Scale Independent Model of the European Innovation System}

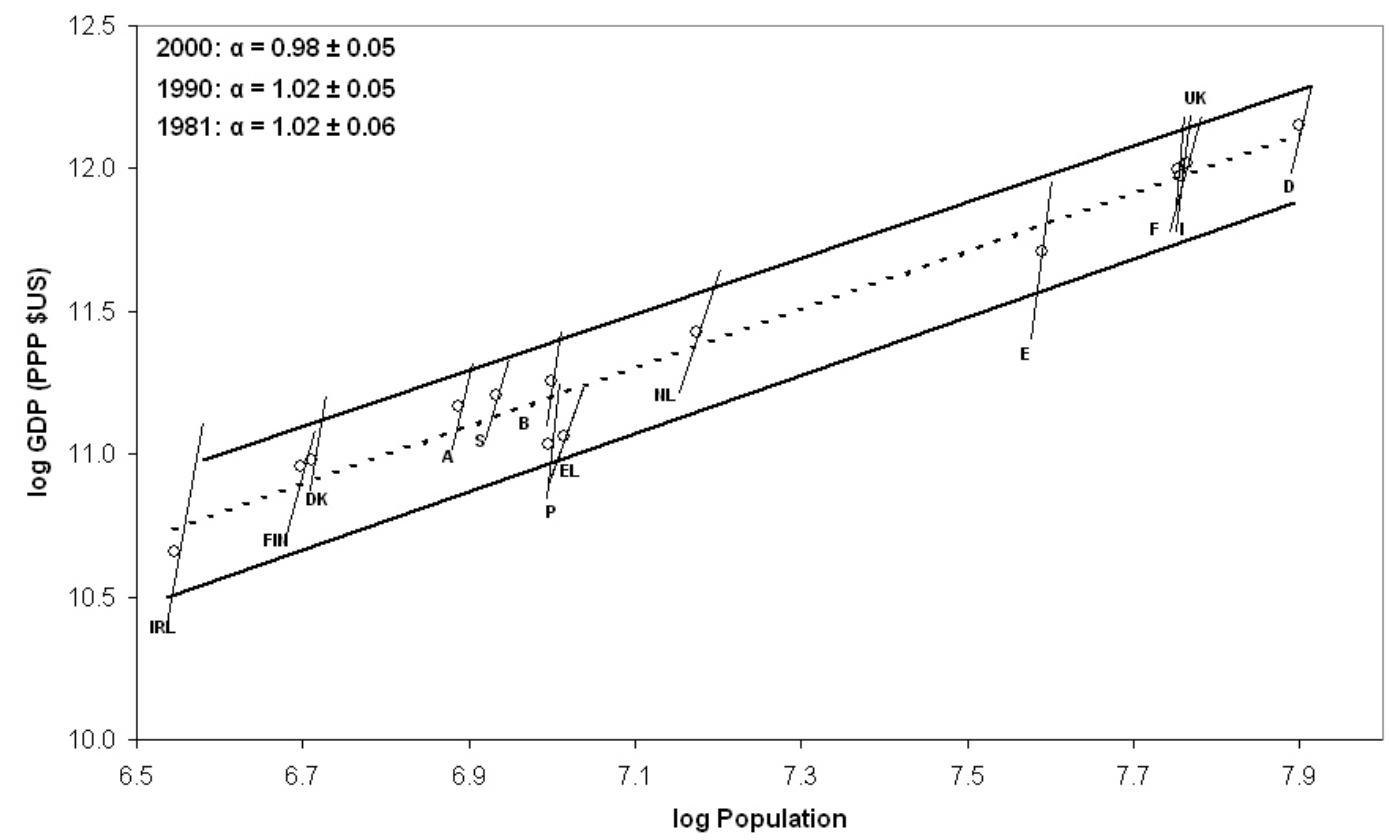

A - Austria, B - Belgium, D - Germany, DK - Denmark, E - Spain, EL - Greece, F - France, FIN - Finland, I - Italy, IRL -Ireland, NL -Netherlands, P - Portugal, S - Sweden and UK -United Kingdom

\section{B. Scale Independent Model of the Canadian Innovation System}

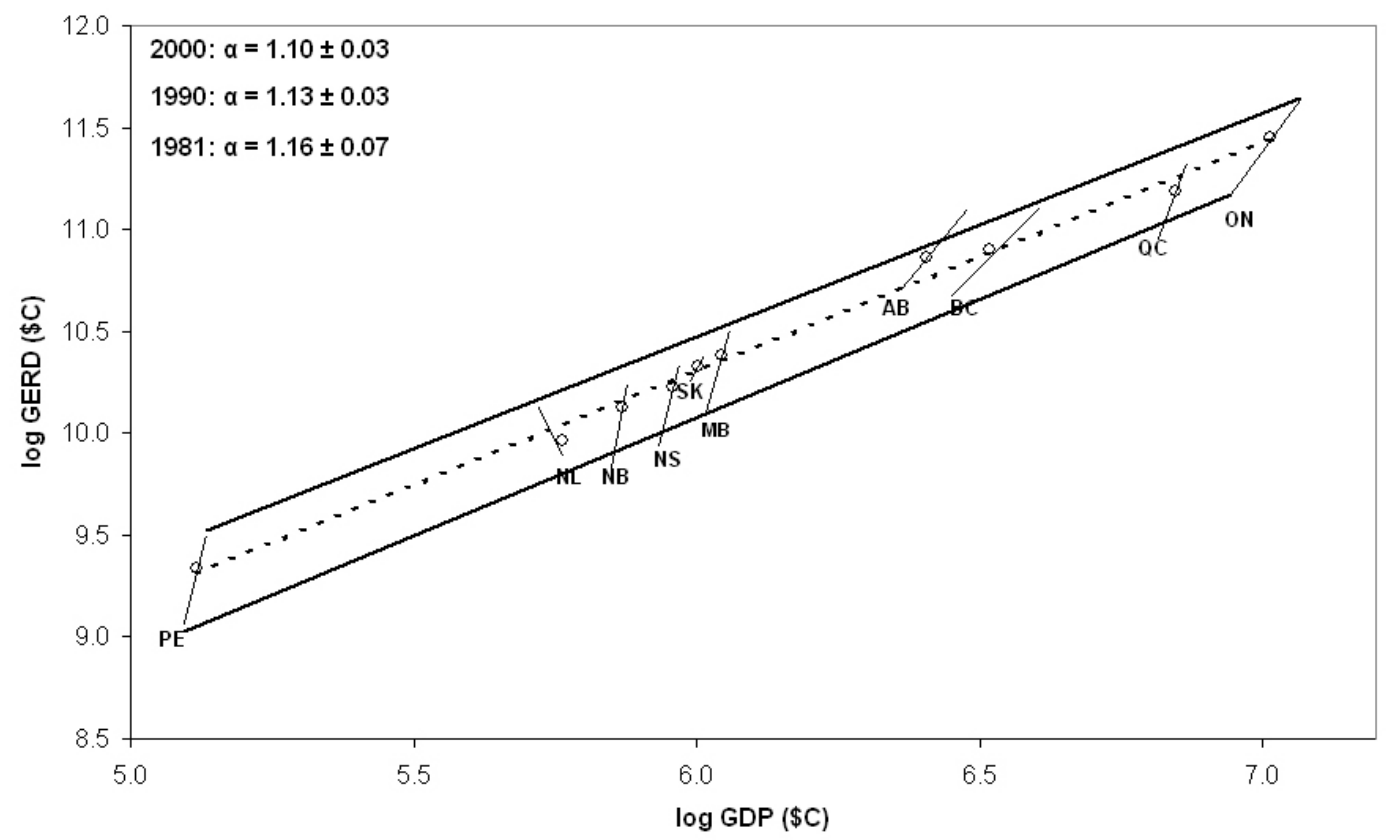

AB - Alberta, BC - British Columbia, MB - Manitoba, NB - New Brunswick, NL -Newfoundland \& Labrador, NS - Nova Scotia, ON - Ontario, PE - Prince Edward Island, QC - Quebec and SK - Saskatchewan

Figure 8 - Scale-independent GDP-population model of (A) Europe and (B) Canada from 1981 to 2000 . 


\section{European and Canadian Innovation Systems}

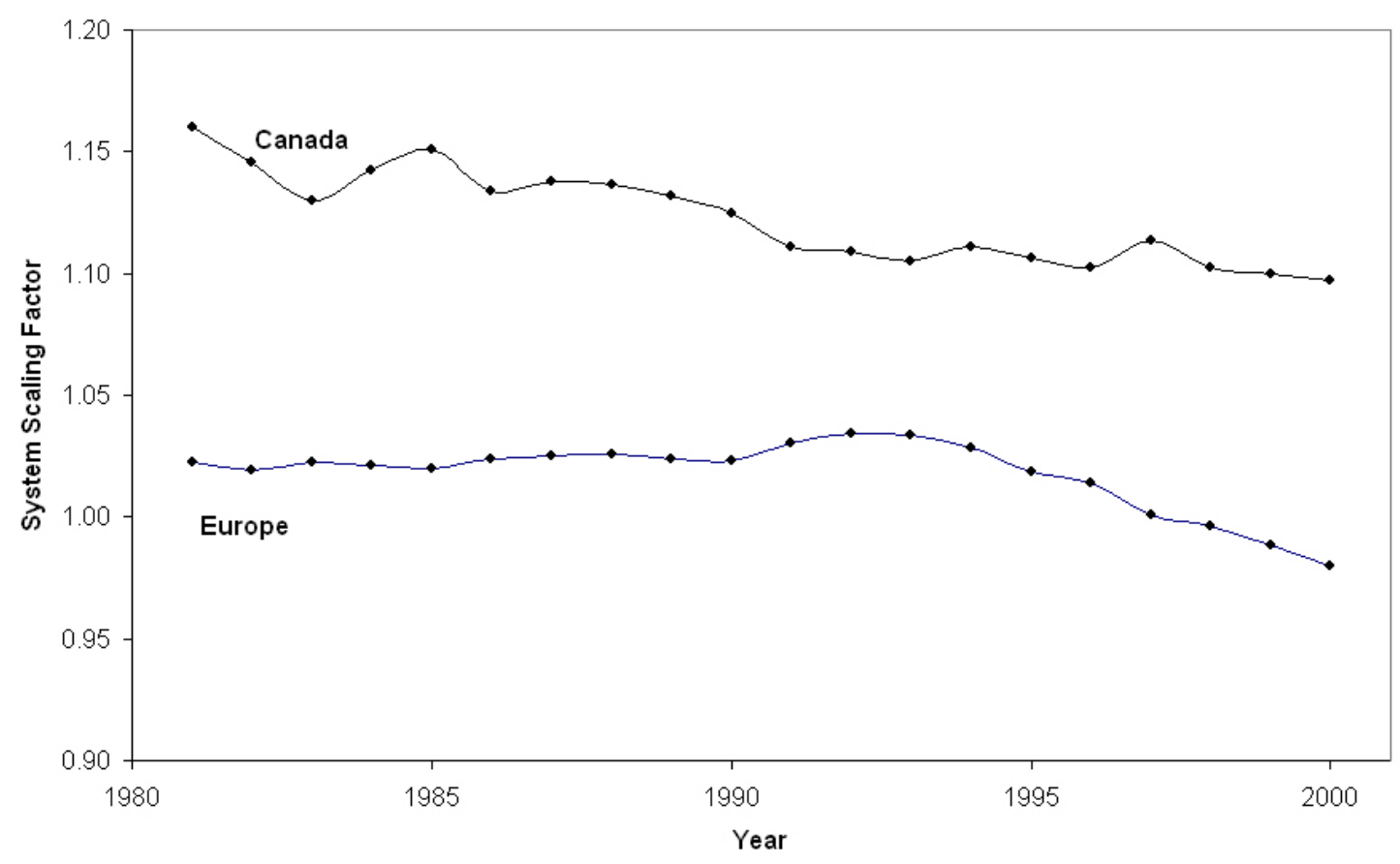

Figure 9 - GDP-Pop systemic scaling factor for the Europe and Canada from 1981 to 2000. 